\title{
RESEARCH
}

Open Access

\section{FAK displacement from focal adhesions: a promising strategy to target processes implicated in cancer progression and metastasis}

Ioanna Antoniades ${ }^{\dagger}$, Maria Kyriakou $^{\dagger}$, Anna Charalambous, Katerina Kalalidou, Andri Christodoulou, Maria Christoforou and Paris A. Skourides ${ }^{*}$ (D)

\begin{abstract}
Background: Focal adhesion kinase (FAK) is a non-receptor tyrosine kinase that is overexpressed or activated in several advanced-stage solid cancers. It is known to play both kinase-dependent and-independent roles in promoting tumor progression and metastasis. Numerous inhibitors, targeting either the enzymatic or scaffolding activities of FAK have been generated, with varying degree of success. Here, we describe a novel approach to sitespecifically target both kinase-dependent and -independent FAK functions at focal adhesions (FAs), the primary sites at which the kinase exerts its activity.

Methods: We took advantage of the well-characterized interactions between the paxillin LD motifs and the FAK FAT domain and generated a polypeptide (LD2-LD3-LD4) expected to compete with interactions with paxillin. Coimmunoprecipitation experiments were performed to examine the interaction between the LD2-LD3-LD4 polypeptide and FAK. The effects of LD2-LD3-LD4 in the localization and functions of FAK, as well as FA composition, were evaluated using quantitative immunofluorescence, cell fractionation, FA isolation and Western Blot analysis. Live cell imaging, as well as 2-D migration and cell invasion assays were used to examine the effects on FA turnover and tumor cell migration and invasion.

Results: Expression of the LD2-LD3-LD4 polypeptide prevents FAK localization at FAs, in a controlled and dosedependent manner, by competing with endogenous paxillin for FAK binding. Importantly, the LD2-LD3-LD4 peptide did not otherwise affect FA composition or integrin activation. LD2-LD3-LD4 inhibited FAK-dependent downstream integrin signaling and, unlike existing inhibitors, also blocked FAK's scaffolding functions. We further show that LD2-LD3-LD4 expression markedly reduces FA turnover and inhibits tumor cell migration and invasion. Finally, we show that dimers of a single motif, linked through a flexible linker of the proper size, are sufficient for the displacement of FAK from FAs and for inhibition of tumor cell migration. This work raises the possibility of using a synthetic peptide as an antimetastatic agent, given that effective displacement of FAK from FAs only requires dimers of a single LD motif linked by a short flexible linker.
\end{abstract}

(Continued on next page)

\footnotetext{
* Correspondence: skourip@ucy.ac.cy

${ }^{\dagger}$ Ioanna Antoniades and Maria Kyriakou contributed equally to this work. Department of Biological Sciences, University of Cyprus, P.O. Box 20537, 2109 Nicosia, Cyprus
} licence and your intended use is not permitted by statutory regulation or exceeds the permitted use, you will need to obtain permission directly from the copyright holder. To view a copy of this licence, visit http://creativecommons.org/licenses/by/4.0/ The Creative Commons Public Domain Dedication waiver (http://creativecommons.org/publicdomain/zero/1.0/) applies to the data made available in this article, unless otherwise stated in a credit line to the data. 
(Continued from previous page)

Conclusion: In conclusion, these results suggest that FAK displacement from FAs is a promising new strategy to target critical processes implicated in cancer progression and metastasis.

Keywords: Focal adhesion kinase (FAK), Paxillin, Focal adhesions, Cell migration, Cell invasion, Cancer

\section{Background}

Cells and their associated microenvironment exhibit a complicated bidirectional communication that is critical for both normal tissue homeostasis and for tumor cell growth, progression and metastasis [1]. The Focal Adhesion Kinase (FAK) has been identified as a critical regulator and signal transducer for these Extracellular Matrix (ECM)-tumor cell interactions [2,3] and has been implicated in many aspects of the metastatic process including adhesion, migration, secretion of MMPs, spindle orientation and invasion [4-7]. FAK has also been found to exhibit altered (usually elevated) expression and/or activation in most human epithelial cancers, resulting in enhanced invasive potential and poor overall patient survival [8]. FAK has therefore, become an attractive target for anti-cancer therapies.

FAK has kinase-dependent and -independent functions, both of which are involved in cancer progression. However, most of the recently developed inhibitors, have focused on blocking the protein's enzymatic activity. These include antibodies [9, 10], dominant negative constructs [11-14] and small molecule inhibitors, that primarily target the ATP binding site, or allosterically inhibit the kinase domain [15], leading to decreased tumor cell viability, growth or apoptosis. However, targeting the kinase domain of FAK has been complicated by the fact that the ATP-binding site shares consensus sequences and structural domains across many different tyrosine kinases, making it less suitable for clinical testing, due to off-target effects [16].

An alternative approach is to inhibit FAK's kinaseindependent activities by blocking specific scaffolding functions of the protein. This has been attempted using peptides, small molecule and antibody inhibitors that disrupt interactions between FAK and various binding partners including VEGFR-3 [17], IFGR1 [18], c-Met [19, 20], Mdm-2 [21] and p53 [22], with variable efficiency.

One of the most highly studied multi-protein complexes, that serve as sites of integration of growth factor signaling and integrin pathways, directing changes as diverse as gene expression and cytoskeletal reorganization, are Focal Adhesions (FAs). The focal adhesion targeting (FAT) domain of FAK is both necessary and sufficient for localization at FAs and facilitates interactions with FAassociated proteins including Paxillin [23, 24], Talin [25, 26], p130Cas [27], Grb2, ASAP1 [28] and p $85 \alpha$ of PI3K [29]. It is a highly conserved four helix bundle with a large hydrophobic core [30, 31]. It includes two surface exposed hydrophobic pockets (HPs), one located at the surface of helices 1 and 4, and the second located at the surface of helices 2 and 3 [32]. These are absolutely essential for FAK targeting to FAs [30, 33, 34]. The best characterized interaction of the FAT HPs is that with the short ( 9 amino acids) Leucine-rich LD motifs of Paxillin [23, 24]. This interaction is important for FAK FA localization, suggesting that Paxillin is one of the major proteins responsible for the kinase's recruitment to these complexes [33-35]. Paxillin itself, is targeted to FAs via the C-terminal LIM (Lin11, Isl-1 \& Mec-3) domains, however the interacting proteins responsible for its localization have not been identified [24, 36]. Once FAK is localized at FAs, it phosphorylates various proteins either directly or through the recruitment of Src kinases [37, 38], leading to efficient integrin signal transduction and cell migration.

The aim of the present study was to develop and test a new strategy for the simultaneous inhibition of both enzymatic and scaffolding functions of FAK, specifically at FAs, by interfering with FAK targeting to these complexes. To do so, we took advantage of the well characterized binding of the LD motifs of Paxillin with the FAK FAT HPs, to prevent interactions with endogenous proteins, responsible for FA targeting. We show that a polypeptide including the LD2 and LD4 motifs of Paxillin, specifically displaces endogenous FAK from FAs in a dose dependent manner without otherwise affecting FA composition or integrin activation. We go on to show that these effects are the result of a competing interaction of the polypeptide with endogenous paxillin, for binding to the FAT domain of FAK. Furthermore, we show that effective displacement of FAK from FAs can be accomplished using dimers of a single LD motif, linked by a short flexible linker. FAK displacement from FAs leads to inhibition of downstream integrin signaling, reduced FA turnover, defects in cell spreading and inhibition of cell migration and invasion. Our findings demonstrate that preventing FAK targeting and functions, specifically at FAs, represents a promising new strategy to prevent molecular and cellular processes implicated in tumor cell metastasis.

\section{Materials and methods}

\section{Plasmids and DNA constructs FLAG LD2-LD3-LD4}

The DNA encoding amino acids 54-279 of human Paxillin, was amplified via PCR using pCS108 GFP LD2- 
LD3-LD4 as template and primers F2 and R2 (Additional File 1: Table S1).

The PCR program was as follows: 2 min at $95^{\circ} \mathrm{C}$ for initial denaturation, followed by 35 cycles of $15 \mathrm{~s}$ at $95^{\circ} \mathrm{C}, 30 \mathrm{~s}$ at $67^{\circ} \mathrm{C}, 1 \mathrm{~min}$ at $68^{\circ} \mathrm{C}$ and final extension at $68^{\circ} \mathrm{C}$ for $10 \mathrm{~min}$.

The PCR product was cloned in frame and downstream to FLAG, in pFLAG CMV-2, using the NotI and EcoRI restriction sites.

\section{GFP LD2-LD3-LD4 and mCherry LD2-LD3-LD4}

The DNA encoding amino acids 54-279 of human Paxillin, was amplified via PCR using pCS2++ GFPPaxillin as template and primers $\mathrm{F} 1$ and R1 (Additional File 1: Table S1).

\section{GFP LD2-LD3-LD4 $\Delta L R$}

The DNA encoding amino acids 139-279 of Paxillin, was amplified via a two-step PCR using GFP LD2-LD3LD4 as template and primers F3, F4 and R2 (Additional File 1: Table S1).

\section{GFP LD2-LD4}

For the generation of GFP LD2-LD4 constructs with linkers of various sizes, multi-step PCRs using the following templates and primers were performed.

GFP LD2-LD4 30aa (referred in the text as GFP LD2LD4): GFP LD2-LD3-LD4 $\triangle \mathrm{LR}$ was used as template and primers F4, R4, R5, R6 and R3 (Additional File 1: Table S1).

GFP LD2-LD4 15aa: GFP LD2-LD4 30aa was used as template and primers F4, R7 and R3 (Additional File 1: Table S1).

GFP LD2-LD4 25aa: GFP LD2-LD4 15aa was used as template and primers F4, R8 and R3 (Additional File 1: Table S1).

\section{GFP LD2}

The DNA encoding amino acids 420-449 of human Paxillin, was amplified via PCR using pCS2++ GFP LD2-LD4 as template and primers F5 and R9 (Additional File 1: Table S1).

\section{GFP LD4}

The DNA encoding amino acids 783-845 of human Paxillin, was amplified via PCR using pCS2++ GFP LD2LD4 as template and primers F6 and R10 (Additional File 1: Table S1).

The PCR program for the above was as follows: $2 \mathrm{~min}$ at $95^{\circ} \mathrm{C}$ for initial denaturation, followed by 35 cycles of $15 \mathrm{~s}$ at $95^{\circ} \mathrm{C}, 30 \mathrm{~s}$ at $67^{\circ} \mathrm{C}, 1 \mathrm{~min}$ at $68^{\circ} \mathrm{C}$ and final extension at $68^{\circ} \mathrm{C}$ for $10 \mathrm{~min}$.

The PCR products of the above were cloned into a pCS108 vector (between NotI and XhoI restriction sites) already including either the EGFP or mCherry gene (between EcoRI and NotI).

\section{GFP LD2-LD2}

A two-step cloning strategy was followed using GFP LD2-LD4 6x short as template.

An LD2-linker fragment was generated using primers F4, R11 and R12 (Additional File 1: Table S1).

The PCR product was cloned in frame and downstream to GFP, in pCS108-GFP using the NotI and XbaI restriction sites.

A linker-LD2 fragment was generated using primers F7, F8 and R13 (Additional File 1: Table S1):

The PCR product was cloned in frame in the pCS108GFP LD2-linker plasmid, using the XbaI and XhoI restriction sites.

\section{GFP LD4-LD4}

A multi-step PCR was performed, using GFP LD2- LD4 as template and primers F9, F10, R14, R15 and R12 (Additional File 1: Table S1):

The PCR program was as follows: $2 \mathrm{~min}$ at $95^{\circ} \mathrm{C}$ for initial denaturation, followed by 35 cycles of $15 \mathrm{~s}$ at $95^{\circ} \mathrm{C}, 30 \mathrm{~s}$ at $67^{\circ} \mathrm{C}, 1 \mathrm{~min}$ at $68^{\circ} \mathrm{C}$ and final extension at $68^{\circ} \mathrm{C}$ for $10 \mathrm{~min}$.

The PCR product was cloned in frame and downstream to GFP, in pCS108-GFP using the NotI and XhoI restriction sites.

\section{pLV-tetO-LD2-LD4.}

The DNA encoding motifs LD2 and LD4 connected with a 30 amino acid-long linker composed of 6 GGGS repeats was amplified via PCR using pCS108 GFP LD2LD4 as template and primers F11 and R16 (Additional File 1: Table S1). The PCR program was as follows: 2 min at $95^{\circ} \mathrm{C}$ for initial denaturation, followed by $35 \mathrm{cy}$ cles of $15 \mathrm{~s}$ at $95^{\circ} \mathrm{C}, 30 \mathrm{~s}$ at $67^{\circ} \mathrm{C}, 1 \mathrm{~min}$ at $68^{\circ} \mathrm{C}$ and final extension at $68^{\circ} \mathrm{C}$ for $10 \mathrm{~min}$. The PCR product was cloned in pLV-tetO-Oct4 vector, using the EcoRI restriction site (to replace Oct4) [39].

pCS2++ TagRFP FAK, pCS108 FusionRed Vinculin and pCS108 RFP Vinculin were generated by replacing the GFP sequence with that of TagRFP or FusionRed in pCS2++ GFP FAK and pCS108 GFP Vinculin respectively [40]. pCS2++ mKate FAK was described elsewhere [34]. pCS2-myc-GFP-dSH2 was obtained from Addgene.

\section{Cells, cell culture and transfection}

HeLa (CCL-2) and MDA MB-231 (HTB-26) cells were obtained from ATCC and were tested for mycoplasma contamination. HeLa and MDA MB-231 cells were maintained in DMEM (Biosera) supplemented with 10\% FBS (Biosera) and 1X Antibiotic-Antimycotic (Gibco). H460 (HTB-177) cells were maintained in RPMI 1640 medium (ThermoFisher Scientific) with 10\% FBS, $1 \mathrm{mM}$ sodium pyruvate (Gibco) and $1 \mathrm{X}$ Antibiotic-Antimycotic. HCT 116 (CCL-247) cells were maintained in McCoy's 5A medium (ThermoFisher Scientific) supplemented with 
10\% FBS and 1X Antibiotic-Antimycotic. Transient transfections with Lipofectamine 2000 (Invitrogen) were performed according to manufacturer's instructions. Cells were observed $24 \mathrm{~h}$ after transfection to verify expression and then used for subsequent experiments.

\section{GFP and GFP LD2-LD4 stable cell line generation}

For the production of infectious viral particles, HEK 293 $\mathrm{T}$ cells were transfected (calcium phosphate) with lentiviral plasmids encoding either a) reverse tetracycline-controlled trans-activator (rtTA-N144) (Addgene), or b) GFP LD2-LD4 (pLV-tetO-LD2-LD4) or c) GFP (pLenti-CMV-GFP-Hygro) (Addgene), together with the packaging (pCMV-dR8.91) and envelope (pCMV-VGV-G) plasmids. Viral supernatants were collected after 48 and $72 \mathrm{~h}$, filtered using a $0.45 \mathrm{~mm}$ syringe and stored at $4^{\circ} \mathrm{C}$.

GFP Hela cells were generated by transduction of pLenti-CMV-GFP-Hygro, while GFP LD2-LD4 Hela cells were generated by co-transduction of pLV-tetOLD2-LD4 and rtTA-N144 viral supernatants, in the presence of $10 \mu \mathrm{g} / \mathrm{ml}$ Polybrene (Merck Millipore). $10 \mu \mathrm{g} / \mathrm{ml}$ doxycycline was added for $48 \mathrm{~h}$ to induce GFP-LD2-LD4 expression [41]. Cells were allowed to recover for $72 \mathrm{~h}$ prior to selection with hygromycin B (Sigma-Aldrich) (400 $\mu \mathrm{g} / \mathrm{ml}$ for 10 days).

\section{Antibodies}

Antibodies used for western blot analysis: mouse antiGFP (1:1000; Proteintech, \#50430-2-AP), rabbit antipFAK Y576 (1:200; Santa Cruz, \#sc-16563), rabbit antipFAK Y397 (1:200; Novus, \#NBPI-60837), rabbit antipPaxillin Y31 (1:200; Santa Cruz, \#sc-14035), mouse anti-FAK (1:1000; Proteintech, \#66258-I-1g), mouse anti-Paxillin (1:5000; BD Biosciences, \#610051), rabbit anti-FLAG (1:1000; Proteintech, \#80010-1-RR).

Antibodies used in immunofluorescence experiments: mouse anti-FAK 4.47 (1:1000; Millipore, \#05-537), mouse anti-FAK (1:1000; Proteintech, \#66258-I-1g), rabbit anti-FAK C-20 (1:200; Santa Cruz, \#sc-558), rabbit anti-pFAK Y576 (1:200; Santa Cruz, \#sc-16563), rabbit anti-pFAK Y397 (1:200; Novus, \#NBPI-60837) goat anti-Talin C-20 (1:750; Santa Cruz, \#sc-7534), mouse anti-Paxillin (1:750; R\&D Systems, \#AF4259), mouse anti-Vinculin (1:1000; Sigma, \#V9131), rabbit anti-Vinculin (1:2000; Proteintech, \#26520-I-AP), mouse anti-av Integrin H-2 (1:100; Santa Cruz, \#sc-376156), rabbit anti-pPaxillin Y31 (1:200; Santa Cruz, \#sc-14035), mouse anti-p-Tyr (pY20) (1:200; Santa Cruz, \#sc-508), rat anti-active $\beta 1$-Integrin 9EG7 (1:700; BD Bioscences, \#550531), mouse anti-p130 Cas 35B.1A4 (1:500; Santa Cruz, \#sc-20029), mouse anti-Tensin C-2 (1:1000; Santa Cruz, \#sc-376367).

\section{Focal adhesion isolation}

The isolation of focal adhesion complexes was based on the protocol of Jones et al. (2015) with some modifications [42]. Briefly, cells were plated on 10-cm cell culture dishes and grown for $48 \mathrm{~h}$ (10 plates per condition). Cells were fixed with $1 \%$ formaldehyde for $5 \mathrm{~min}$ at room temperature and formaldehyde was quenched with $0.125 \mathrm{M}$ glycine for $10 \mathrm{~min}$. Cells were washed with ice cold PBS and incubated with modified RIPA buffer for 5 min on ice. Cell bodies were removed with highpressure water and the remaining focal adhesion were collected by scraping in Laemmli sample buffer.

\section{Cell Lysis, Western blot and Immunoprecipitation}

For western blot analysis cells were rinsed with ice-cold PBS and lysed in RIPA buffer supplemented with protease inhibitors (2x Halt Protease inhibitor cocktail, Thermo Scientific) and sodium orthovanadate $(5 \mathrm{mM}$, Sigma) for $10 \mathrm{~min}$. Lysates were cleared by centrifugation $\left(15,000 \mathrm{~g}, 4{ }^{\circ} \mathrm{C}\right.$ for $\left.10 \mathrm{~min}\right)$ and $40 \mu \mathrm{g}$ of extracted protein were used for Western blot analysis as previously described [34].

For GFP or FLAG pull-down, cells from two $10-\mathrm{cm}$ plates for each condition, were lysed in $1.5 \mathrm{ml}$ lysis buffer $(20 \mathrm{mM}$ Tris $\mathrm{pH} 7.5,150 \mathrm{mM} \mathrm{NaCl}, 1 \% \mathrm{v} / \mathrm{v}$ NP40) supplemented with protease inhibitors and sodium orthovanadate. Cleared protein extracts were gently mixed with $5 \mu \mathrm{l}$ of GFP-Trap Agarose beads (Chromotek) or $30 \mu \mathrm{l}$ anti-FLAG M2-Agarose affinity gel (Sigma Aldrich), overnight at $4{ }^{\circ} \mathrm{C}$. The beads or gel were washed 3 times with lysis buffer and then resuspended in $50 \mu \mathrm{l} 2 \mathrm{x}$ Laemli sample buffer (Bio-Rad) supplemented with 2mercaptoethanol (Fluka). Samples were boiled for $10 \mathrm{~min}$ at $95^{\circ} \mathrm{C}$ and the supernatant containing the immunocomplexes was collected by centrifugation $\left(2500 \mathrm{~g}, 4^{\circ} \mathrm{C}\right.$ for 5 min) and analysed by western blot.

For FAK immunoprecipitation, five $10-\mathrm{cm}$ plates of GFP, uninduced GFP LD2-LD4 or induced LD2-LD4 cells were lysed in $1.5 \mathrm{ml}$ lysis buffer (as described above). Protein extracts were incubated with $10 \mu \mathrm{g}$ of anti-FAK antibody (Proteintech), overnight at $4{ }^{\circ} \mathrm{C}$ (extracts of uninduced GFP LD2-LD4 cells incubated with no antibody were used as a negative control.). Extracts were then incubated with $30 \mu \mathrm{l}$ Protein-G Sepharose CL$4 \mathrm{~B}$ beads (GE Healthcare) for $2 \mathrm{~h}$ at $4{ }^{\circ} \mathrm{C}$. Beads were washed 5 times with lysis buffer and boiled in $40 \mu \mathrm{l} 2 \mathrm{x}$ Laemli sample buffer and analysed by western blot.

\section{Inhibitor treatment}

Cells were incubated at $37^{\circ} \mathrm{C}$ with either $50 \mu \mathrm{M}$ Chloropyramine hydrochloride (C4) inhibitor (Santa Cruz Biotechnology) for $24 \mathrm{~h}$, or $10 \mu \mathrm{M}$ PF228 inhibitor (Santa Cruz Biotechnology) for $3 \mathrm{~h}$. 


\section{Immunofluorescence}

Cells $\left(\sim 7 \times 10^{4}\right)$ seeded on $\mathrm{HCl}$-charged glass coverslips $(15 \mathrm{~mm})$, were PFA or methanol/acetone fixed and immunostained as described elsewhere [40]. Briefly, cells were rinsed with cold PBS and fixed with 4\% PFA for 10 min, followed by quenching with $10 \mathrm{mM}$ Glycine (Sigma) for $10 \mathrm{~min}$ and permeabilization with $0.2 \%$ Triton X-100 (Bio-Rad) for $10 \mathrm{~min}$. Alternatively cells were fixed with cold methanol/acetone (1:1) for $20 \mathrm{~min}$ at $20^{\circ} \mathrm{C}$. Cells were then blocked with $10 \%$ donkey serum (Jackson Immumoresearch) in 1X PBS for $30 \mathrm{~min}$ and incubated with primary antibodies for $1 \mathrm{~h}$. This was followed by several washes in $1 \mathrm{X}$ PBS and incubation with secondary antibodies for $1 \mathrm{~h}$. Cells were thoroughly washed with 1X PBS and mounted in ProLong Diamond antifade mountant (Molecular Probes).

\section{Invasion assay}

25-30,000 MDA MB-231 cells, transiently transfected with LD2-LD3-LD4, were resuspended in a solution of $30 \%$ Matrigel (Corning), $0.02 \%$ Hoechst (Invitrogen) and $1 \%$ FBS in DMEM (final volume: $20 \mu \mathrm{l}$ ) and placed as a droplet on a rectangular chambered coverslip $(24 \times 60 \mathrm{~mm})$, previously treated with organosilane (RainX). A round $10 \mathrm{~mm}$ coverslip, with $2 \mathrm{~mm}$ spacers was placed on top, flattening the droplet into a round disc. The Matrigel was allowed to set in a humidifying chamber $\left(37^{\circ} \mathrm{C}\right.$ ) for $30 \mathrm{~min}$. A second layer of $30 \%$ Matrigel (with 5\% FBS) containing 0,02\% fluorescent beads (Molecular Probes) (final volume: $50 \mu \mathrm{l}$ ), was added under the round coverslip, to occupy the area surrounding the disc and allowed to set as described above. The chambered rectangular coverslip was then filled with DMEM (containing 10\% FBS). The setup was used for time-lapsed imaging of cell invasion over a period of 48-72 h. Alternatively, it was used to obtain static images after 48 or $72 \mathrm{~h}$.

\section{Imaging}

Confocal imaging was performed on a Zeiss LSM 710 laser scanning confocal microscope (Carl Zeiss AG, Germany), with a Plan-Apochromat $63 x / 1.40$ oil DIC immersion objective using lasers $488 \mathrm{~nm}, 543 \mathrm{~nm}$ and $633 \mathrm{~nm}$. Super-resolution imaging was performed on a Zeiss LSM 900 laser scanning confocal microscope with Airyscan 2 (Carl Zeiss AG, Germany) with a PlanApochromat $63 \mathrm{x} / 1.40$ oil DIC immersion objective using lasers $475 \mathrm{~nm}, 555 \mathrm{~nm}, 630 \mathrm{~nm}$. Widefield fluorescence imaging was performed on a Zeiss Axio Imager Z1 microscope (Carl Zeiss AG, Germany), with a PlanApochromat $63 x / 1.40$ oil $\mathrm{Ph} 3$ immersion objective. Live cell migration imaging was performed on a Zeiss Axio Imager Z1 microscope and an Axiovert 200 motorized inverted microscope (Carl Zeiss AG, Germany), with a
Plan-Apochromat10x/0.4 Ph1 objective. Western blot imaging was performed using a UVP Biospectrum imaging system.

Image analysis was performed using Zen 2010 and Axiovision 4.8 software (Carl Zeiss AG, Germany) and Adobe Photoshop (Adobe Inc., San Jose CA) to adjust brightness and contrast. Figures were constructed with Adobe Photoshop.

\section{Quantification}

VisionWorks software (UVP LLC, CA) was used for the quantification of western blot results using raw data from non-processed images for densitometry analysis.

Imaris image analysis software (Oxford Instruments, UK) and Zen 2010 software (Carl Zeiss AG, Germany) were used for the quantification of fluorescence intensities. Classification was according to GFP expression levels and was performed by determining the median GFP intensity of expressing cells in each experiment and comparing this to the GFP intensity of individual cells from the same experiment. Non-expressing cells were used as negative controls and were intrinsic to each experiment, since transient transfections never lead to $100 \%$ transfection efficiency.

To quantify FA-localization of specific proteins, cells were stained for the protein of interest as well as an additional FA marker, shown to retain its localization upon LD2-LD3-LD4 expression (i.e. vinculin or talin). Individual FAs were automatically selected, based on the staining of the unaffected FA marker, and the mean cytosolic intensity of each protein was subtracted from that on individually selected FAs, thus enabling specific protein quantification on individual FAs. To quantify the localization of the Src SH2 domain at FAs, cells were transfected with GFP Src dSH2 alone or together with mCherry LD2-LD3-LD4. Cells were stained for Vinculin and individual FAs were selected based on Vinculin staining. FA localization was then assessed as described above. For the quantification of FA turnover rates, time-lapse images of cells expressing RFP Vinculin (used as an FA marker) were aligned in ImageJ (NIH, USA) and analyzed. Individual FAs were manually selected (ROI) at time point t0 and the mean fluorescence intensity of each ROI was documented for each time point of the recording $(\mathrm{t} 0=0 \mathrm{~min}, \mathrm{t} 1=$ $5 \mathrm{~min}, \mathrm{t} 2=10 \mathrm{~min}, \mathrm{t} 3=15 \mathrm{~min}, \mathrm{t} 4=20 \mathrm{~min}, \mathrm{t} 5=25$ $\mathrm{min})$. The mean cytosolic intensity for each time point was subtracted from that of each ROI.

Quantification of cell migration rates was performed using the Imaris image analysis software and performing automatic tracking of individual cells. The migration efficiency was calculated as the percentage change of the migration rate of GFP expressing cells, compared to that of non-expressing control cells. 
Quantification of cell invasion efficiency was performed with the Imaris image analysis software, using static images of cells at the end of $72 \mathrm{~h}$. Total cell numbers were determined by automatic selection of individual nuclei (stained with Hoechst). LD2-LD3-LD4 expressing cell numbers were determined by automatic selection of GFP positive cells. Control cell numbers were determined by subtracting GFP positive cell numbers from total cell numbers. Invading cell numbers were determined by automatic detection of cells within a specific ROI. ROIs included areas defined by a fluorescently delineated boundary between a fluorescentlylabeled low-serum concentration gel and a non-labeled high-serum concentration gel. The invasion efficiency was calculated as the percentage of cells that traversed the boundary.

\section{Statistical analysis}

Graph generation and statistical analysis were performed using Prism software (GraphPad, San Diego, CA). All graph data are shown as mean values while error bars represent S.E.M. Statistical analysis was performed using two-tailed unpaired t-tests with 95\% confidence interval. For experiments examining FAs, all statistical analysis was performed on total FA numbers from all cells considered. All experiments have been performed at least 3 times.

\section{Results}

Using the Paxillin LD motifs to interfere with interactions targeting FAK to FAs, as a strategy to inhibit FAK

Our main aim was to develop and test a new strategy that would block both enzymatic and scaffolding functions of FAK, specifically at FAs, as a possible new approach for FAK inhibition. To achieve this, we set out to interfere with interactions responsible for FA targeting. The FAT domain is both necessary and sufficient to drive FAK at FAs. Previous work from our group and others revealed that the two hydrophobic pockets (HPs) formed by the FAT domain are essential for FA targeting $[33,34,43]$. The best characterized interaction of the HPs is with the LD2 and LD4 motifs of Paxillin [30, 35]. We postulated that a peptide encoding these LD motifs, but lacking FA targeting sequences (LIM domains), would interfere with interactions responsible for FAK FA targeting. We also took into account the fact that several cancer-linked Paxillin mutations have been mapped to the intrinsically disordered regions between LDs and not on the motifs themselves, such as P30S, G105A and A127T that lie between LD1 and LD2 and P233L and T255I that lie between LD3 and LD4 [44, 45]. Given the significance of this intermediate linking region, in LD interactions with binding partners and in LD scaffolding functions, we decided that it should be included in the construct [46]. We therefore generated a construct containing LD2-LD3-LD4 and intermediate linking regions, fused to GFP, hereunto referred to as LD2-LD3-LD4 (Fig. 1a). This construct led to expression of a stable protein, at the expected molecular weight, which localized primarily in the cytosol (Fig. 1b and c).

We went on to examine if LD2-LD3-LD4 interacted with FAK, in co-immunoprecipitation experiments, using extracts of HeLa cells transiently transfected with GFP LD2-LD3-LD4 or GFP (negative control). As shown in Fig. 1d, a band corresponding to co-precipitated FAK (at $125 \mathrm{kD}$ ), was detected only in the precipitates from cells expressing GFP LD2-LD3-LD4, showing that LD2LD3-LD4 specifically interacts with FAK, as expected. Overall, these experiments show that LD2-LD3-LD4 interacts with FAK directly and given its cytosolic localization it could potentially prevent FAK localization at FAs.

\section{Expression of LD2-LD3-LD4 leads to the specific and dose-dependent displacement of FAK from FAs}

In order to examine if LD2-LD3-LD4 expression could specifically disrupt FAT domain interactions and displace FAK from FAs, HeLa cells were transiently transfected with LD2-LD3-LD4, seeded on FN coated coverlips for two hours, fixed and immuno-stained for FAK and Talin. Talin was selected as a stable marker of mature FAs, given the fact that its recruitment to the complex relies on direct binding to $\beta$ integrin cytoplasmic tails. As shown, expression of LD2-LD3-LD4 led to the clear displacement of FAK from FAs, while Talin localization was unaffected (Fig. 2a). This effect was confirmed using a second FAK antibody and, in addition, exogenous mKate FAK (Additional File 2: Fig. S1 a-c). We quantified this displacement, by calculating the ratio of FAK in the cytosol to FAK at FAs, revealing that LD2-LD3-LD4 expression led to a 4-fold reduction of FA-localized FAK (Fig. 2b). Interestingly, a similar quantification for Talin showed that LD2-LD3-LD4 expression leads to an increase in FA localized Talin, possibly due to enlargement of the FA complexes (Fig. 2c). In order to account for this, we calculated the FAK to Talin ratio at FAs, which revealed a dramatic 5-fold reduction in LD2-LD3-LD4 expressing cells, suggesting that LD2LD3-LD4 is very effective in displacing FAK from FAs (Fig. 2d), unlike expression of GFP, which was used as a negative control (Additional File 2: Fig. S1d). We then examined how the levels of LD2-LD3-LD4 affected displacement efficiency, and revealed a clear dose response relationship; in cells expressing relatively high levels of GFP, we observed complete loss of FAK from FAs while in cells expressing moderate or low levels of GFP, we could still detect FAK at FAs, albeit at significantly reduced levels (Fig. 2e). 


\section{a}

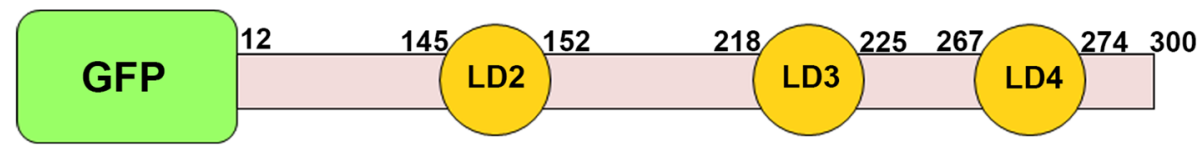

b

GFP LD2-LD3-LD4

C

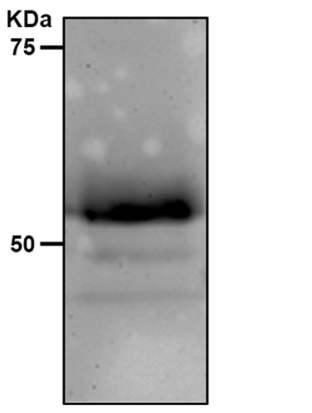

GFP LD2-LD3-LD4

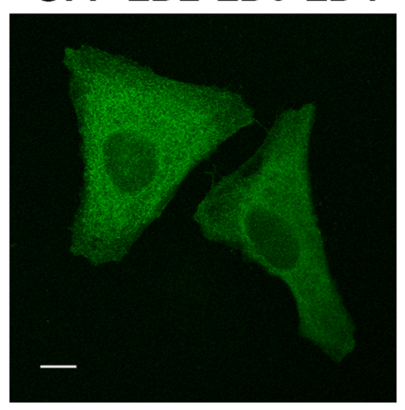

d
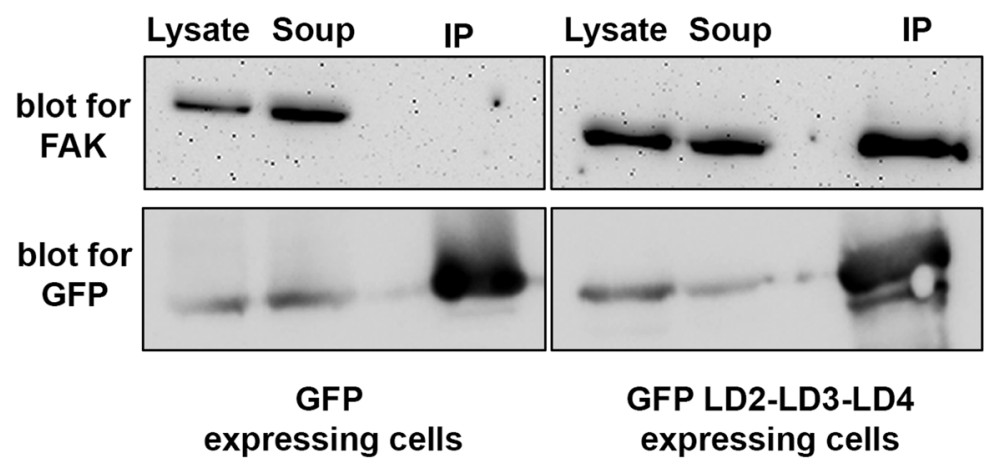

Fig. 1 LD2-LD3-LD4 interacts with FAK directly a) Schematic representation of the LD2-LD3-LD4 polypeptide, composed of amino acids 54-279 of Paxillin, including the LD2-LD3-LD4 motifs and fused to GFP. b) Representative Western Blot (using anti-GFP) showing expression of a stable protein encoding GFP-fused LD2-LD3-LD4, in HeLa cells (expected molecular weight 51 kDa). c) Confocal images of PFA-fixed HeLa cells expressing GFP LD2-LD3-LD4. The protein is primarily localized in the cytosol (Scale bar: $10 \mu \mathrm{m})$. d) Western blots showing immunoprecipitated GFP (left) and GFP LD2-LD3-LD4 (right), blotted for GFP and FAK. Co-precipitation of FAK (125 kDa) is only observed in HeLa cells co-expressing GFP LD2-LD3-LD4

Fluorescent proteins, despite mutations to reduce their ability to dimerize, still maintain some capacity to do so. Additionally, given their globular nature and relatively large size $(27 \mathrm{kD})$ they tend to stabilize fused peptides. Furthermore, GFP displays inherent accumulation to the nucleus and could thus be influencing peptide function, by affecting cellular distribution. To ensure that the LD2-LD3-LD4 peptide is stable and can be used effectively in the absence of GFP, we generated a FLAGtagged peptide which is much smaller in size $(1 \mathrm{kD})$. As shown in Fig. S1e and S1f, the FLAG-tagged peptide can efficiently interact with FAK and lead to effective displacement from FAs., This confirms that the LD2-LD3-
LD4 is sufficiently stable and functional in the absence of a large globular protein.

A previously generated inhibitor of the interaction of FAK with VEGFR3 (C4), was also reported to displace FAK from FAs [47]. This interaction, as characterized by docking studies, takes place through binding of $\mathrm{C} 4$ to His 1025 on Helix 4 of the FAT domain of FAK, adjacent to HP1, to which it may sterically hinder access [17]. We thus decided to compare the efficiency of $\mathrm{C} 4$ with that of LD2-LD3-LD4, to displace FAK from FAs. We examined the distribution of FAK and Talin in HeLa cells, following treatment with high concentrations of C4 $(50 \mu \mathrm{M})$ for $48 \mathrm{~h}$. Surprisingly, C4, failed to visibly 


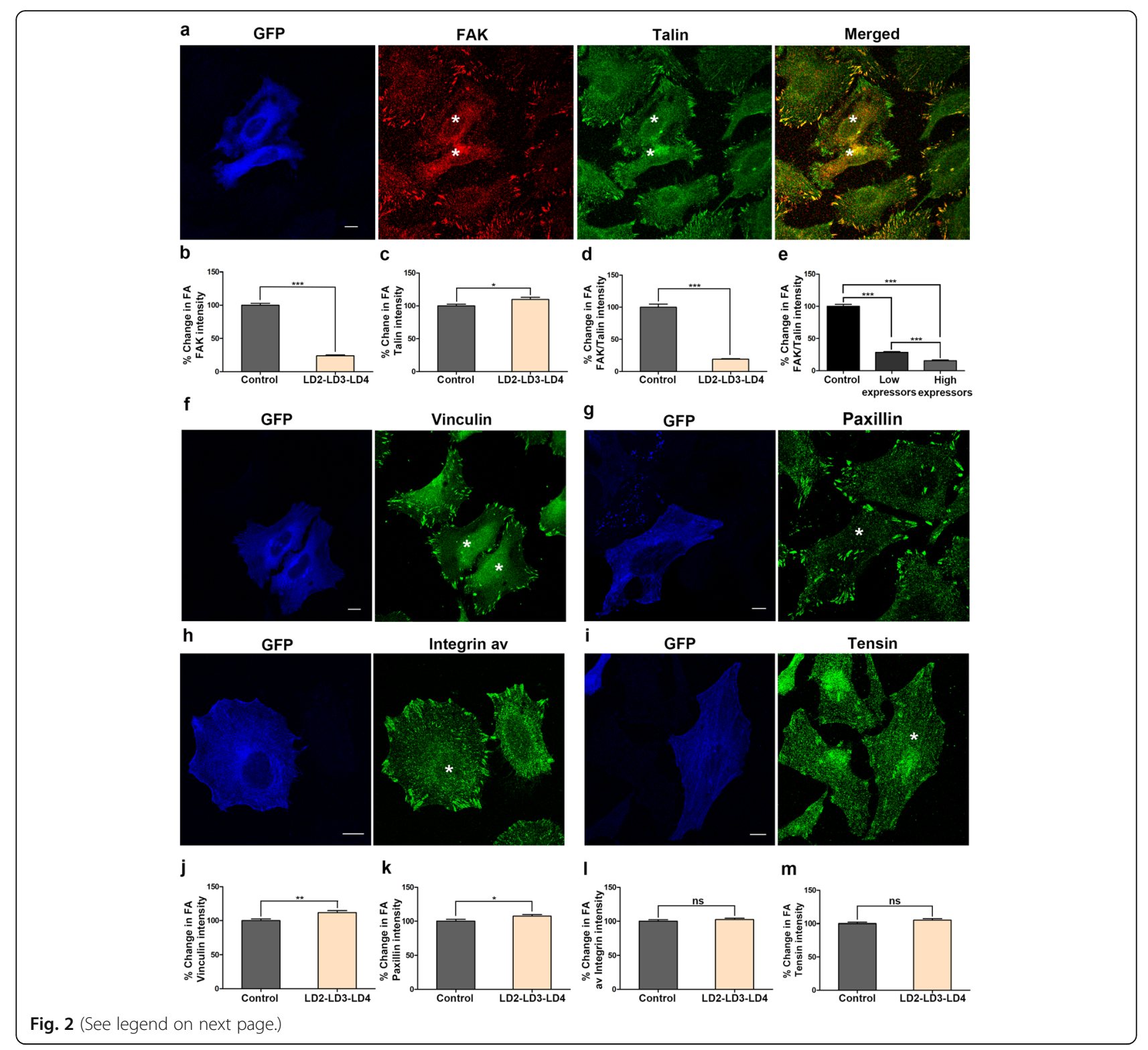


(See figure on previous page.)

Fig. 2 Expression of LD2-LD3-LD4 leads to the dose-dependent displacement of FAK from FAs without affecting overall FA composition a) Confocal images of methanol/acetone fixed HeLa cells, transiently transfected with GFP LD2-LD3-LD4 and immunostained for FAK and Talin. In control cells, FAK strongly localizes at FAs, labeled with Talin. In cells expressing LD2-LD3-LD4 (marked with asterisk) there is no detectable enrichment of FAK at FAs. In contrast, Talin localization at FAs is unaffected. $\mathbf{b}-\mathbf{c}$ ) Quantification of the \% change in the mean FA/cytosolic FAK (b) and Talin (c) intensity ratios. FA/Cytosolic ratio of FAK in control cells is $\sim 4.2$ fold higher (100 $\pm 2.86, n=469$ FAs from 30 cells) compared to cells expressing GFP LD2-LD3-LD4 (23.78 $\pm 1.25, n=409$ FAs from 30 cells), indicating that LD2-LD3-LD4 leads to the displacement of FAK from FAs. In contrast, the FA/cytosolic ratio of Talin is higher in cells expressing GFP LD2-LD3-LD4, possibly due to increased FA size (109.7 \pm 3.22 in expressing, compared to $100 \pm 2.59$ in controls). d) Quantification of the \% change in the mean FAK/Talin intensity (based on $\mathbf{b}$ and $\mathbf{c}$ ) reveals a $\sim 5$-fold drop of FA localized FAK in cells expressing GFP LD2-LD3-LD4 (19.01 \pm 0.91 in expressing, compared to $100 \pm 4.8$ in control cells). e) Dose-dependent displacement of FAK from FAs as indicated by quantification of the \% change in the mean FAK/Talin intensity in control compared to expressing cells (high and low) $(15.55 \pm 1.25, n=188$ FAs from 13 cells, expressing higher, and 28.52 $\pm 1.14, n=218$ FAs from 17 cells, expressing lower amount of GFP LD2-LD3-LD4, compared to $100 \pm 3.1, n=229$ FAs from 28 control cells). To discriminate between high and low expressing cells, we initially determined the mean GFP intensity of all expressing cells, and then compared this to the GFP intensity of individual cells, so as to classify them as high or low expressors. (f-i) Confocal images of PFA-fixed control and GFP LD2-LD3-LD4 (marked with asterisk) HeLa cells immunostained for Vinculin (f), Paxillin (g), av. Integrin (h) and Tensin (i) showing that localization of these proteins is not affected by the expression of GFP LD2-LD3-LD4. (j-m) Corresponding quantification of the \% change in the mean FA/cytosolic intensity for each protein presented in $\mathrm{f}-\mathrm{i}$. FA/cytosolic ratio of Vinculin (j) and Paxillin (k) is higher in cells expressing GFP LD2-LD3-LD4, possibly due to increased FA size $(111.8 \pm 2.86, n=460$ FAs from 30 expressing, compared to $100 \pm 2.57, n=455$ FAs from 30 control cells for Vinculin; $107.5 \pm 2.29, n=402$ FAs from 30 expressing, compared to $100 \pm 2.64, n=432$ FAs from 30 control cells for Paxillin). There is no significant difference in the FA/ cytosolic ratio of av. Integrin (I) and Tensin (m) (102.5 $\pm 1.94, n=568$ FAs from 30 expressing, compared to $100 \pm 2.16, n=485$ FAs from 30 control cells for av. Integrin; $105 \pm 2.24, n=532$ FAs from 30 expressing, compared to $100 \pm 2.09, n=568$ FAs from 30 control cells for Tensin). Scale bars: $10 \mu \mathrm{m}$. The error bars represent standard error of the mean (S.E.M). ${ }^{* *} ; p<0.0001,{ }^{* *} ; p<0.005,{ }^{*} ; p<0.05$

displace FAK from FAs, compared to controls. Quantification of FAK to Talin signal ratios on FAs of control and treated cells, confirmed that C4 did not affect FAK FA localization. However, after inhibitor treatment, some cells appeared to have smaller FAs, with low FAK and Talin signals, because they were detaching from the substrate. These data show that $\mathrm{C} 4$ does not specifically block FAK targeting to FAs and suggest that in order to efficiently displace the protein, disruption of interactions taking place at the HPs is necessary (Additional File 2: Fig. S1g).

Given the potent displacement of FAK from FAs induced by LD2-LD3-LD4, we wanted to examine the specificity of this effect and possible consequences on FA composition. We therefore examined the localization of additional core FA proteins including Integrins (av), Paxillin, Tensin and Vinculin. As shown, similarly to Talin, FA localization of these proteins was not reduced by LD2-LD3-LD4 expression, suggesting that the effect of LD2-LD3-LD4 is specific to FAK and that the composition of FA complexes is broadly unaltered in expressing cells (Fig. 2f-m). Overall, these data provide evidence that LD2-LD3-LD4 could serve as an effective, sitespecific inhibitor of interactions at the HP sites within the FAT domain of FAK and prevent FAK localization at FAs in a dose-dependent manner.

\section{LD2-LD3-LD4 inhibits both kinase-dependent and scaffolding functions of FAK at FAs}

FAK is a major transducer of integrin signaling and becomes phosphorylated and activated in response to integrin-dependent adhesion. Given that LD2-LD3-LD4 interacts with FAK directly, leading to its displacement from FAs, we went on to address its effects on FAK activation. To do so, we examined the phosphorylation state of a) Tyr397, the major FAK auto-phosphorylation site required for activation, b) Tyr576, which resides in the activation loop of the kinase domain and has been shown to lead to full activation upon phosphorylation and c) paxillin Tyr31, one of the major FAK/Src downstream targets $[37,48]$. As shown, LD2-LD3-LD4 expression led to a significant reduction of phosphorylation at these sites, suggesting that LD2-LD3-LD4 expression blocks FAK activation and downstream signaling (Fig. 3a). Importantly, this reduction becomes even more significant, since transient transfection efficiency is never $100 \%$ and thus what we observe represents an underestimation of the effect. In order to examine the effects of LD2-LD3-LD4 on FAK phosphorylation in individual cells, we carried out Immunofluorescence (IF) using phospho-specific antibodies. As shown, LD2-LD3-LD4 expression led to a dramatic drop in FAK phosphorylation (on Tyr397) at FAs, suggesting that it effectively eliminates FAK activation at these complexes (Additional File 2: Fig. S1h).

One of the best characterized downstream targets of FAK is Paxillin, which becomes phosphorylated on Tyrosines 31 and 118, in response to integrin activation in wild type but not in FAK null cells [49]. We thus went on using quantitative immunofluorescence and calculated the ratio of phosphorylated-Paxillin (pPax) to Paxillin, in order to assess the effects of LD2-LD3-LD4 on Paxillin phosphorylation, specifically at FAs. As shown, expression of LD2-LD3-LD4 led to a significant reduction of the levels of pPax, suggesting that it not only blocks FAK activation but also downstream 

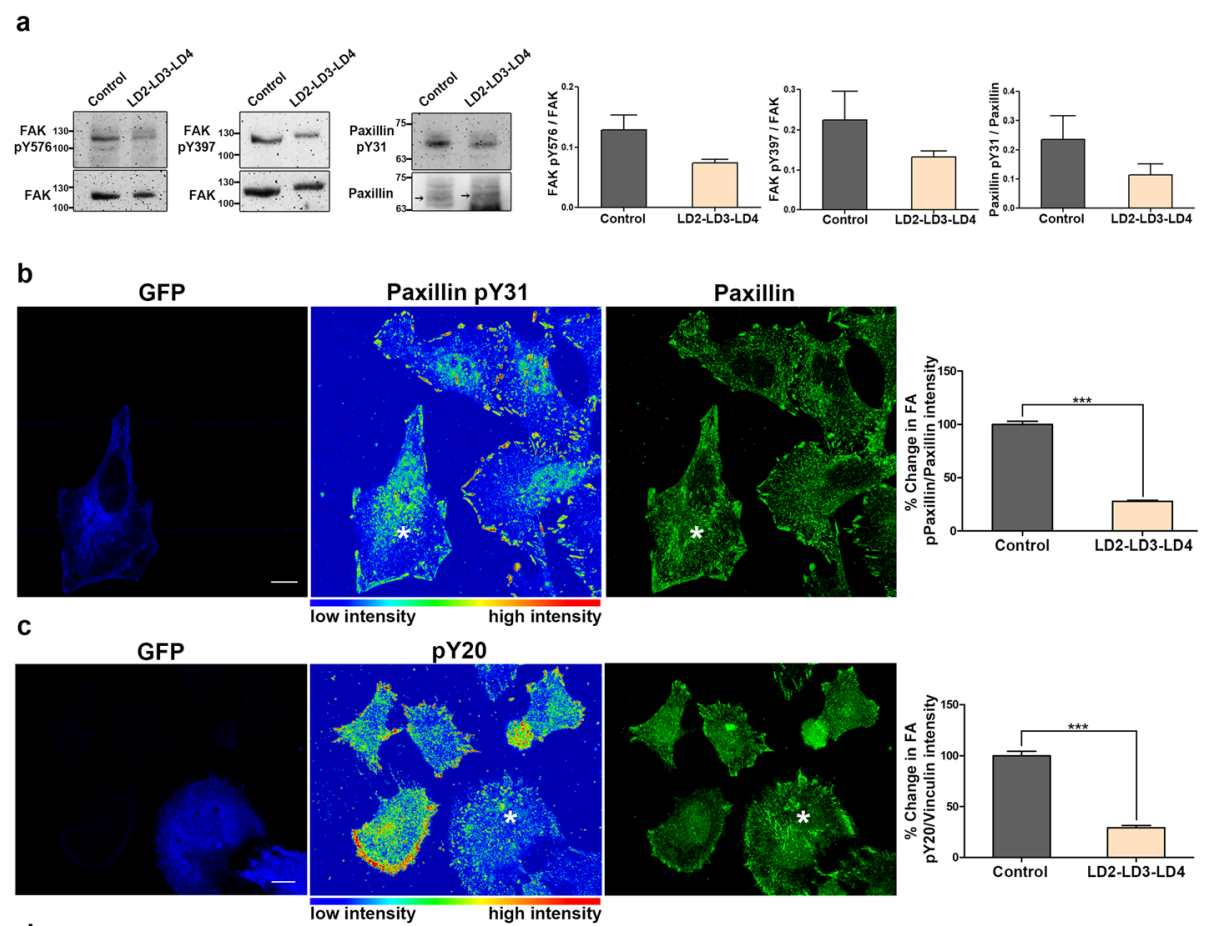

d

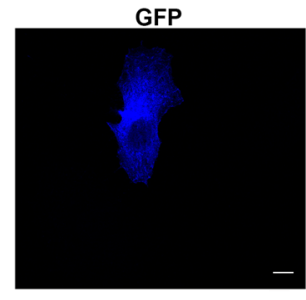

Active $\beta 1$ integrin
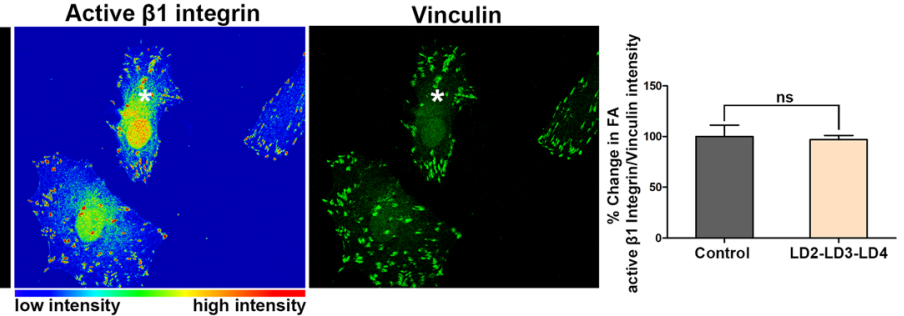

Fig. 3 Expression of LD2-LD3-LD4 blocks kinase-dependent functions of FAK, downstream of integrin activation a) Representative Western Blots and quantification from control cells and cells expressing GFP LD2-LD3-LD4, indicating the phosphorylation status of FAK Tyr 397 and Tyr 576 and Paxillin Tyr 31. Quantification of the ratio of phosphorylated FAK over total FAK shows reduction of the phosphorylation at both Tyr 576 $(0.13 \pm 0.024$ in control, $0.074 \pm 0.005$ in GFP LD2-LD3-LD4 expressing samples) and Tyr 397 (0.22 \pm 0.0073 in control, $0.13 \pm 0.015$ in GFP LD2-LD3LD4 expressing samples). Paxillin Tyr 31 phosphorylation is also reduced, as indicated by the ratio of phosphorylated over total paxillin (0.24 \pm 0.08 in control, $0.11 \pm 0.04$ in GFP LD2-LD3-LD4 expressing samples). b) Confocal images of PFA-fixed HeLa cells transfected with GFP LD2-LD3-LD4 and immunostained for Paxillin and phosphorylated Paxillin (pY31). Phosphorylated Paxillin signal in control and GFP LD2-LD3-LD4 expressing cells (marked with asterisk) is presented in the middle panel as an intensity color-coded image. Quantification of the \% change in the mean intensity of phosphorylated (pPaxillin) to total Paxillin reveals significant reduction of Paxillin phosphorylation at FAs in cells expressing GFP LD2LD3-LD4 (27.93 $\pm 0.83, n=331$ FAs from 24 cells) compared to control cells (100 $\pm 2.92, n=371$ FAs from 25 cells). c) Confocal images of PFA-fixed HeLa cells transfected with GFP LD2-LD3-LD4, immunostained against phosphorylated tyrosine (pY20) and Vinculin. Intensity of tyrosine phosphorylation in control and GFP LD2-LD3-LD4 expressing cells (marked with asterisk) is presented in the middle panel in a color-coded image. Quantification of the \% change in the mean pY20/Ninculinintensity reveals a 3.4-fold decrease in total phosphorylation at FAs in cells expressing GFP LD2-LD3-LD4 (29.29 $\pm 2.12, n=414$ FAs from 30 cells) compared to control cells ( $100 \pm 4.44, n=435$ FAs from 30 cells). d) Confocal images of PFA-fixed cells transfected with GFP LD2-LD3-LD4 and immunostained against active $\beta 1$ Integrin and Vinculin. Active $\beta 1$ Integrin signal in control and GFP LD2-LD3-LD4 expressing cells (marked with asterisk) is presented in the middle panel as an intensity color-coded image. Quantification of the $\%$ change in the mean active $\beta 1$ Integrin/Vinculin intensity shows that expression of GFP LD2-LD3-LD4 does not affect integrin activation at FAs (100 $\pm 11.18, n=346$ FAs from 30 control, compared to $97.10 \pm 3.98, n=457$ FAs from 30 expressing cells). Scale bars: $10 \mu m$. The error bars represent standard error of the mean (S.E.M). ${ }^{* *} ; p<0.001$

signaling from FAs (Fig. 3b). In agreement with this result, staining of LD2-LD3-LD4 expressing cells with a well characterized pY antibody (pY20) revealed that overall tyrosine phosphorylation is dramatically reduced at FAs, suggesting that signaling is impaired due to FAK displacement (Fig. 3c). Given the dramatic reduction of tyrosine phosphorylation at FAs, we examined whether LD2-LD3-LD4 somehow prevents integrin activation. Quantification of the ratio of active Integrin $\beta 1$ to Vinculin at FAs showed that LD2-LD3-LD4 has no effect on 
integrin activation (Fig. 3d). Therefore, the above data clearly show that LD2-LD3-LD4 expression blocks FAK kinase-dependent signal transduction events, downstream of integrin activation.

Upon recruitment at FAs, FAK is auto-phosphorylated on Tyr397, creating a high-affinity binding site for the SH2 domain of Src, which further phosphorylates FAK on Tyr576 and Tyr577 within the activation loop, leading to maximal enzymatic activity [50]. In order to examine the effects of LD2-LD3-LD4 expression on FAK-mediated Src recruitment to FAs we expressed the SH2 domain of Src fused to GFP (GFP Src_dSH2), previously shown to be necessary and sufficient for FA targeting of Src [51]. As expected, given the previous data indicating FAK FA displacement and abolishment of Tyr397 phosphorylation, expression of LD2-LD3-LD4 led to a significant reduction in Src_dSH2 FA localization, indicating an inability of Src to target FAs (Fig. $4 \mathrm{a}$ and $\mathrm{b}$ ).

FAK also has well-established scaffolding functions, including a kinase independent role in the recruitment of the FAK-Src substrate, p130Cas to FAs [27]. This is achieved through an SH3-dependent interaction with the $\mathrm{C}$ terminal proline-rich regions of FAK [27]. In order to determine if, unlike kinase inhibitors, LD2-LD3-LD4 could also suppress kinase-independent, scaffolding functions, we examined p130Cas localization. LD2-LD3LD4 expressing and control cells, as well as cells treated with a previously characterized FAK kinase inhibitor (PF228) [52], were immunostained for Talin and p130Cas. There was a visible reduction of FA-localized p130Cas in LD2-LD3-LD4 expressing cells, unlike control and PF228-treated cells in which no change was observed as confirmed by quantification of the ratio of p130Cas to Talin (Fig. 4c and d). As expected PF228 treatment led to a clear reduction of tyrosine phosphorylated FAK at FAs but did not interfere with its localization; thus, as expected, p130Cas is maintained at the complex (Additional File 2: Fig. S2a-c). The above results show that unlike inhibitors of FAK's enzymatic activity, expression of LD2-LD3-LD4 blocks both kinasedependent and independent functions at FAs.

\section{Expression of LD2-LD3-LD4 affects FA dynamics, and inhibits migration and invasion of tumor cells}

It is well established that FAK is a critical regulator of FA assembly and disassembly, processes that are fundamental for efficient, directional cell migration [53-55]. Given that expression of LD2-LD3-LD4 displaces FAK from FAs, we initially examined whether this would elicit changes in FA dynamics. For this purpose, we evaluated the number and size of FAs in LD2-LD3-LD4 expressing vs control HeLa cells that were seeded on glass coveslips for $12 \mathrm{~h}$. As shown in Fig. 5a, control cells formed a characteristic pattern of FAs, mainly found at the cell periphery. In contrast, LD2-LD3-LD4 expressing cells displayed a significant increase in both the number and size of FAs with prominent ventral FAs (Fig. 5b and c). This result, suggests a defect in FA turnover and is consistent with previous findings in FAK -/- cells [53]. We went on to directly examine the effects of LD2-LD3LD4 expression on FA turnover. HeLa cells were transfected with RFP-Vinculin alone or co-transfected with RFP-Vinculin and LD2-LD3-LD4, seeded on fibronectincoated chambered slides and time-lapse sequences were recorded, over a period of $35 \mathrm{~min}$. Cells expressing the construct displayed markedly slower FA turnover compared to control cells (Fig. 5d and e). Therefore, these data show that LD2-LD3-LD4 expression elicits defects in FA turnover, leading to the appearance of more and larger FAs, in a similar manner to defects reported in FAK null fibroblasts [53].

Given the central role of FA turnover in cell migration, we decided to examine how LD2-LD3-LD4 affected cell spreading and migration. Control and LD2-LD3-LD4 expressing HeLa cells were seeded on fibronectin-coated coverslips and monitored using time-lapse video microscopy over a period of $16 \mathrm{~h}$. We used a motorized stage to image multiple areas simultaneously, so as to record and track large numbers of cells. Analysis of the recordings revealed that LD2-LD3-LD4 elicited dosedependent defects in both cell spreading and migration (Fig. 6a and b and Additional File 3: Movie S1). In addition, analysis of the time-lapse images revealed that cells expressing high levels of LD2-LD3-LD4, displayed slightly increased apoptosis $(16,9 \%$ compared to $6,2 \%$ in control cells). Similar effects were observed in other highly migratory and metastatic cell lines, namely MDA MB-231 (Additional File 2: Fig. S3a), H460 (Additional File 2: Fig. S3b) and HCT-116 (Additional File 2: Fig. S3c), in which FAK is effectively displaced from FAs, upon expression of LD2-LD3-LD4 (Additional File 2: Fig. S3d-f). Overall, these data show that LD2-LD3-LD4, not only elicits defects in cell spreading and FA turnover, consistent with phenotypes observed in FAK null cells, but is also an effective inhibitor of two-dimensional (2-D) cell migration.

Although active cell migration is a prerequisite for metastasis, there is strong evidence suggesting that 3-D culture and gel invasion assays better mimic the tumor microenvironment and predict therapeutic responses, in vivo, more accurately $[56,57]$. To examine the effects of LD2-LD3-LD4 on tumor cell invasion, we developed a modified Boyden-chamber gel invasion assay, which allows live and end-point evaluation of cell invasion and permits imaging, tracking and quantification of both invading and non-invading cells. The highly invasive MDA MB-231 cells were used for these experiments and both 
a

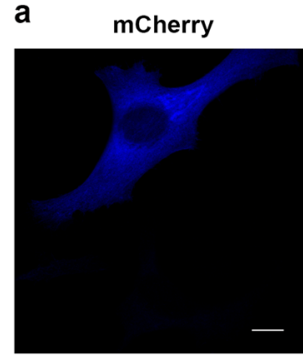

\section{GFP Src dSH2}
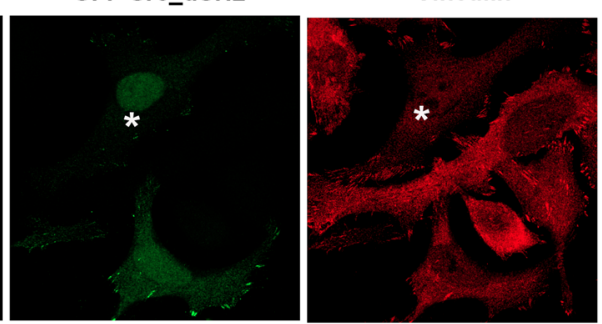

b

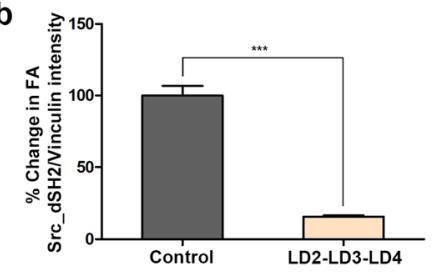

C
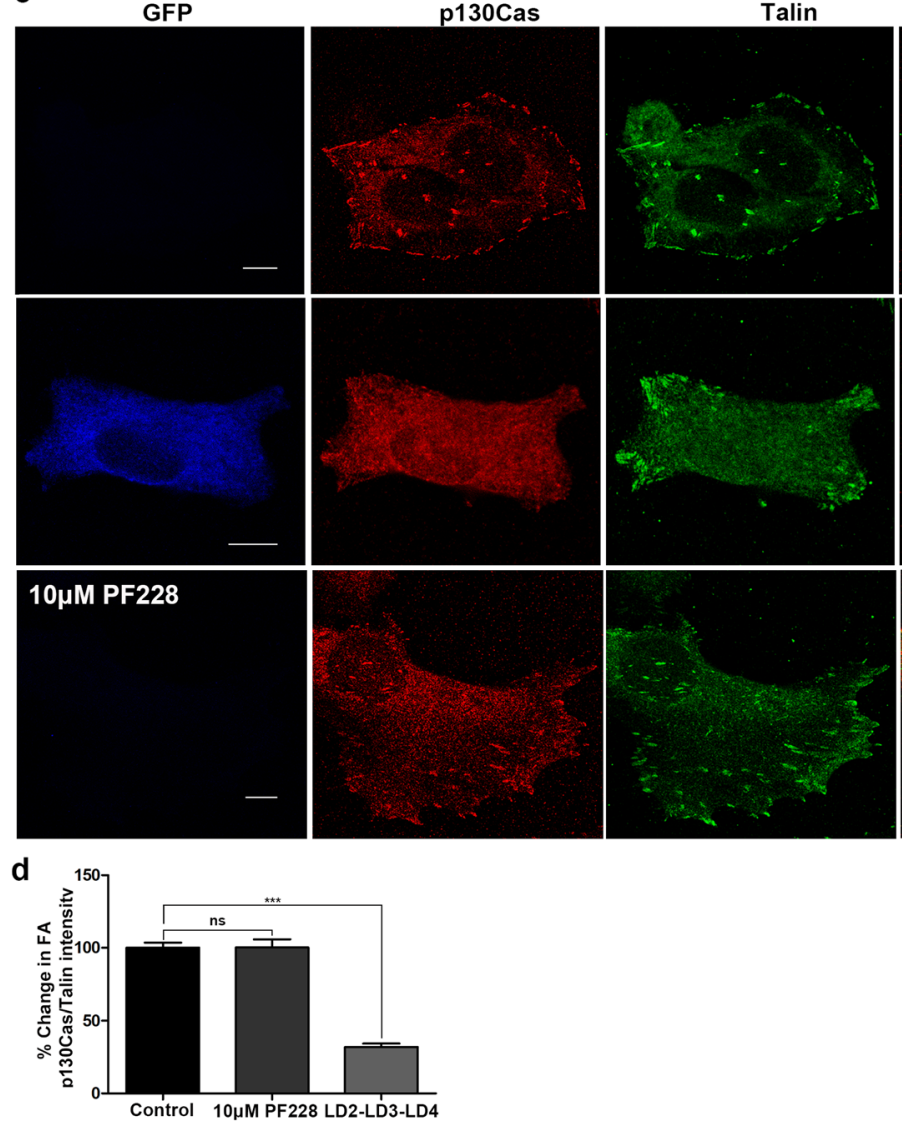

Fig. 4 Expression of LD2-LD3-LD4 blocks kinase-independent, scaffolding functions of FAK at FAs a-b) Super resolution images and quantification of the FA-localization of GFP Src_dSH2 in control (GFP Src_dSH2 only) and mCherry LD2-LD3-LD4 expressing cells (marked with asterisk). Cells were fixed with PFA and immunostained for Vinculin. Expression of mCherry LD2-LD3-LD4 leads to 84.3\% decrease in the SrC_dSH2Ninculin ratio compared to control cells (100 $\pm 6.68, n=419$ FAs from 24 control, 15.67 $\pm 0.93, n=373$ FAs in 22 mCherry LD2-LD3-LD4 expressing cells). c-d ) Confocal images (c) and quantification (d) of control (top panel), LD2-LD3-LD4 expressing (intermediate panel) and PF228 treated (bottom panel) cells, fixed with methanol/acetone and immunostained for p130Cas and Talin. Expression of LD2-LD3-LD4 leads to a 68\% decrease in the p130Cas/Talin ratio compared to control cells, whereas treatment with PF228 does not elicit any significant change $(100 \pm 3.56, n=505$ FAs from 33 control, $31.89 \pm 2.46, n=564$ FAs from 35 LD2-LD3-LD4 expressing and 100.2 $\pm 5.6, n=515$ FAs from 30 PF228-treated cells). F Scale bars: $10 \mu \mathrm{m}$. The error bars represent standard error of the mean (S.E.M). ${ }^{* * *} ; p<0.0001$ 
a
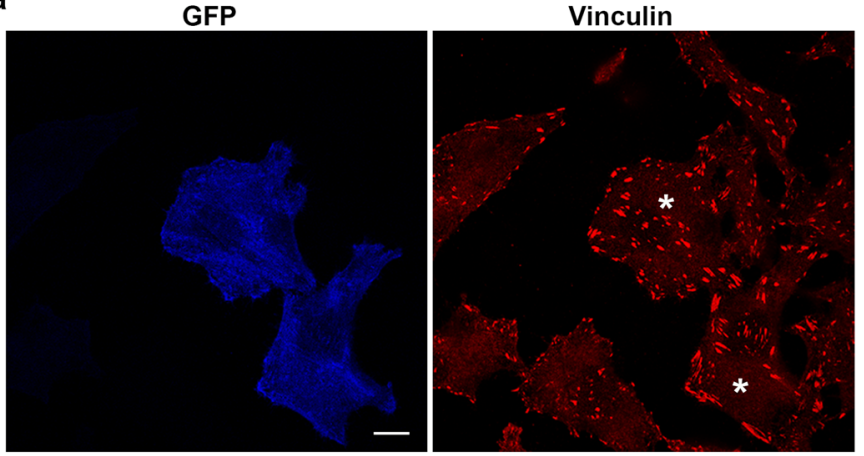

b

C
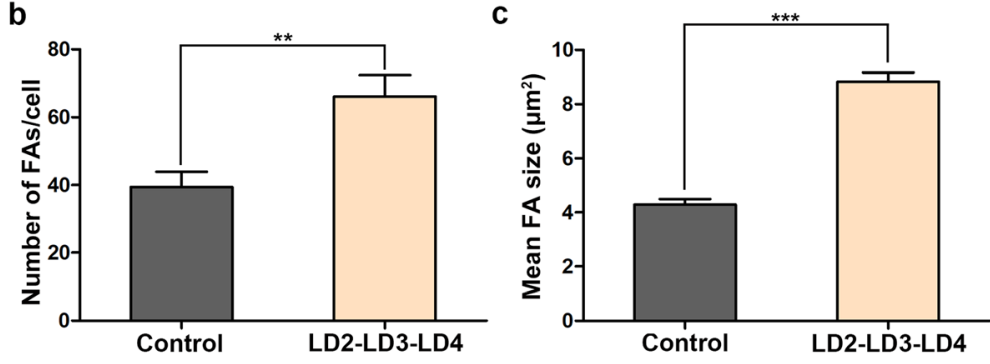

d
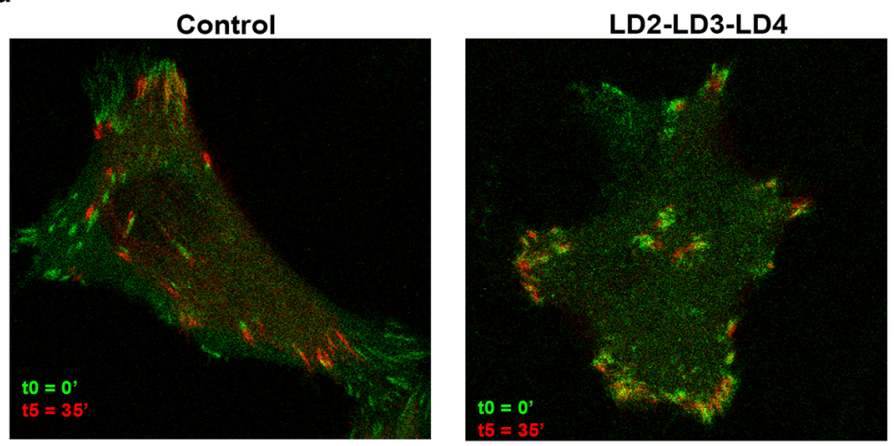

e

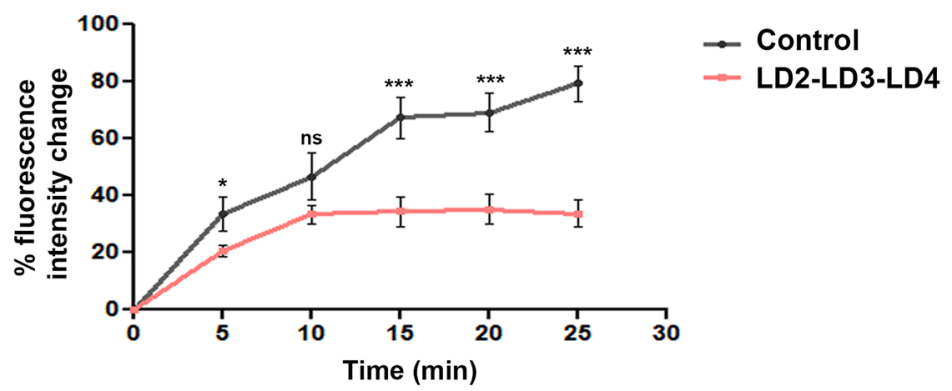

Fig. 5 Expression of LD2-LD3-LD4 leads to an increase in number and size of FAs and a reduction of FA turnover a) Confocal images of PFA-fixed HeLa cells transfected with GFP LD2-LD3-LD4 and immunostained for Vinculin. Cells expressing GFP LD2-LD3-LD4 (marked with asterisk) form more and larger FAs, that are localized more ventrally. (b-c) Quantification of the total number of FAs per cell (b) and mean FA size (c), confirm significant increase of FA number (39.41 $\pm 4.48 \mathrm{FAs}, n=27$ control cells compared to $66.17 \pm 6.35 \mathrm{FAs}, n=29$ GFP LD2-LD3-LD4 expressing cells) and size $\left(4.29 \pm 0.21 \mathrm{~m}^{2}, n=526\right.$ FAs from 27 control cells, compared to $8.83 \pm 0.33 \mu \mathrm{m}^{2}, n=915$ FAs from 29 GFP LD2-LD3-LD4 expressing cells) and. d) Overlayed confocal images from time lapse recordings, showing FA turnover in control and GFP LD2-LD3-LD4 expressing cells. Cells were imaged over a period of $25 \mathrm{~min}$ and FAs were visualized using RFP Vinculin. e) Quantification of the FA turnover rate assessed as the percentage change in the average intensity of selected FAs over time. FAs in GFP LD2-LD3-LD4 expressing cells display significantly slower turnover rates (33.77\% 4.86 change at $25 \mathrm{~min}, \mathrm{n}=52 \mathrm{FAs})$ compared to control cells $(79.39 \% \pm 6.22$ change at $25 \mathrm{~min}, n=38 \mathrm{FAs}) .{ }^{* *} ; p<0.005,{ }^{* * *} ; p<0.0001,{ }^{*} ;$ $p=0.0118,{ }^{* * *} ; p \leq 0.0005$ 


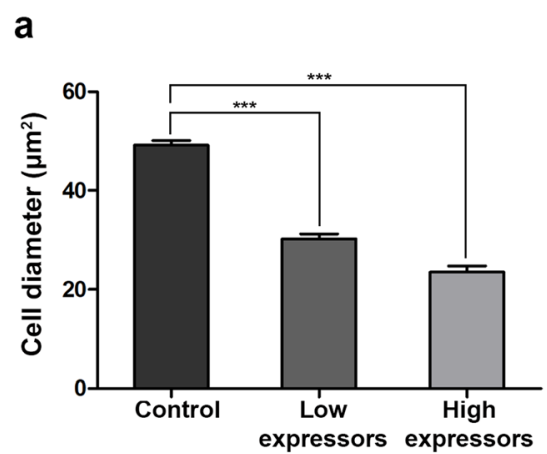

b

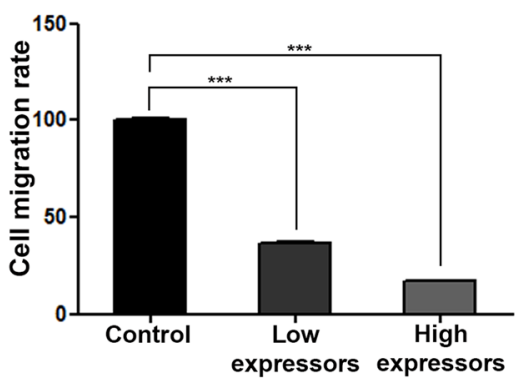

C

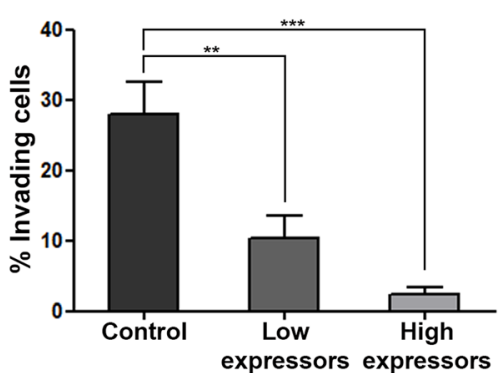

Fig. 6 LD2-LD3-LD4 expression inhibits tumor cell spreading, migration and invasion in a dose-dependent manner a) Expression of GFP LD2-LD3LD4 leads to dose-dependent defects in Hela cell spreading. Spread area is reduced by 38.4 and $52.2 \%$ in low-and high-expressing cells respectively $(49.16 \pm 0.97 \mu \mathrm{m}, n=216$ control cells, compared to $30.27 \pm 0.92 \mu \mathrm{m}, n=102$ low-expressing cells and $23.52 \pm 1.28 \mu \mathrm{m}, n=67$ highexpressing cells). Cell spreading was calculated as a function of the diameter of the attached area on fibronectin coated coverslips, $1 \mathrm{~h}$ after seeding. b) Expression of GFP LD2-LD3-LD4 leads to dose-dependent defects in migration of HeLa. The rate of migration is reduced by 63 and $83 \%$ in low and high-expressing cells respectively, following $16 \mathrm{~h}$ of recording $(100 \pm 1.32, n=549$ in control, compared to $36.62 \pm 0.28, n=263$ in low-expressing and $17.03 \pm 0.2, n=227$ in high-expressing cells). c) Expression of GFP LD2-LD3-LD4 leads to a dose-dependent reduction in the capacity of MDA-MB231 cells to invade Matrigel, as indicated by quantification, using Hoechst staining to detect all cells and GFP to determine expressors. Invasion efficiency is reduced by 63 and $92 \%$ in low-and high-expressing cells respectively, following $72 \mathrm{~h}$ of incubation $(27.95 \pm 4.5 \%$, $n=22,718$ control, compared to $10.39 \pm 03.06 \%, n=8950$ low-expressing and $2.3 \pm 1.02 \%, n=5930$ high-expressing cells). Invasion efficiency is calculated as the percentage of cells traversing a fluorescently delineated boundary from a low-to high-serum concentration gel, in a chemotactic gradient. To discriminate between high and low expressing cells, we initially determined the mean GFP intensity of all expressing cells, and then compared this to GFP intensity of individual cells, so as to classify them as high or low expressors. ${ }^{* *} ; p<0.0001,{ }^{* *} ; p<0.005$

end-point measurements, as well as time-lapse recordings were generated with mixed populations of LD2LD3-LD4 expressing and control cells, in the same setup. As shown, LD2-LD3-LD4 expression, inhibited invasion of MDA MB-231 cells in a dose-dependent manner; in fact, high expression drastically reduced invasion of this highly metastatic cell line (Fig. 6c and Additional File 4: Movie S2). These results show that displacement of FAK from FAs is an effective strategy to block both cell migration, as well as tumor cell invasion. It could therefore form the basis for the development of antimetastatic drugs.

\section{The LD2 and LD4 motifs are sufficient for effective FAK displacement from FAs}

Previous work revealed that LD2 and LD4 are responsible for the interaction with the HPs of FAK [30, 35]. In the work described above, the construct used to displace FAK from FAs also contained LD3, as well as intermediate linking regions (Fig. 1a). This was initially deemed necessary given the significant regulatory role assigned to these unstructured linking segments for the FAKpaxillin interaction [44-46]. However, these regions also bare numerous phosphorylation sites and binding sites for proteins other than FAK, thus their presence would be expected to be detrimental to the specificity of the polypeptide and lead to off target effects. In addition, the large size of the polypeptide containing these regions (24 kDa, 226aa) poses restrictions in its potential use as a metastatic inhibitor, in the form of a synthetic peptide since it is well beyond the size limit for effective peptide synthesis (100-120aa). Therefore, we decided to determine the minimum paxillin sequences required for efficient displacement of FAK from FAs. To this end we implemented a subtractive approach, removing individual linking regions in a stepwise fashion and assessing the activity of each construct.

We initially deleted the region upstream of LD2 (LD1LD2 linking region-LR), previously reported to be necessary for optimal binding to FAK [23, 24, 30] and examined how it affected the peptide's capacity to displace FAK from FAs (Fig. 7a). This construct led to expression of a stable polypeptide, at the expected molecular weight, hereunto referred as LD2-LD3-LD4 $\triangle \mathrm{LR}$ (Fig. 7b). As shown, expression of LD2-LD3-LD4 $\triangle \mathrm{LR}$ led to clear displacement of FAK from FAs, while Vinculin localization (used as an FA marker) was unaffected as expected (Fig. 7c). Quantification of the FAK to Vinculin ratio showed that LD2-LD3-LD4 $\triangle \mathrm{LR}$ displaced FAK with the same efficiency as the original peptide, suggesting that the linking segment upstream of LD2 does not play a pivotal role for efficient binding of the LD2 and LD4 motifs to the FAT HPs in the cell (Fig. 7d).

Next, we went on to examine whether the intermediate linking region between LD2 and LD4, containing the LD3 motif, plays a role in the ability of the polypeptide to displace FAK. Using the DNA encoding for LD2LD3-LD4 $\triangle \mathrm{LR}$ as template, we replaced the region between the LD2 and LD4 motifs with a flexible standard linker (GGGGS). Optimization of the length of the 


\section{a}

LD2-LD3-LD4 $\Delta L R$
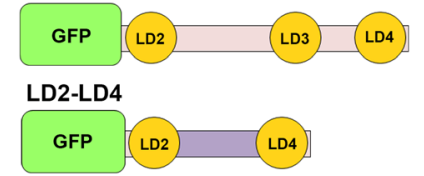

C
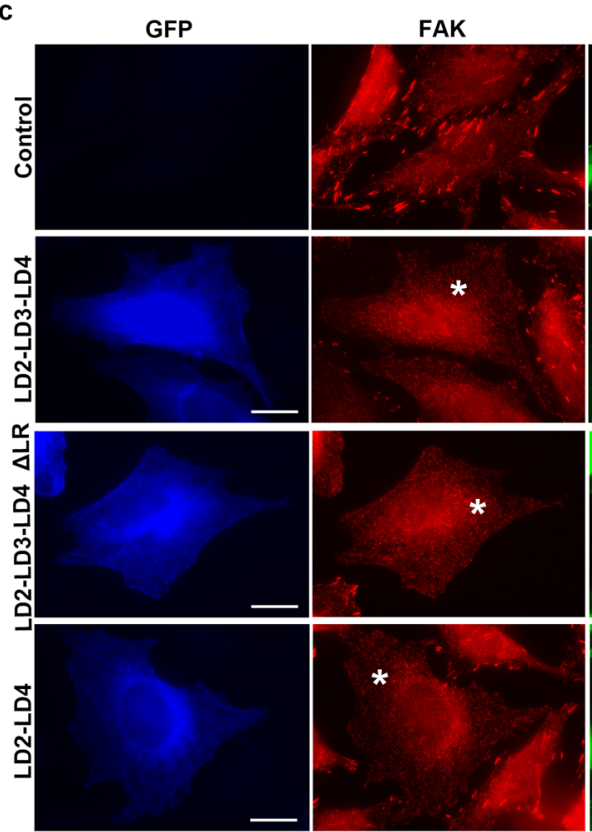

d

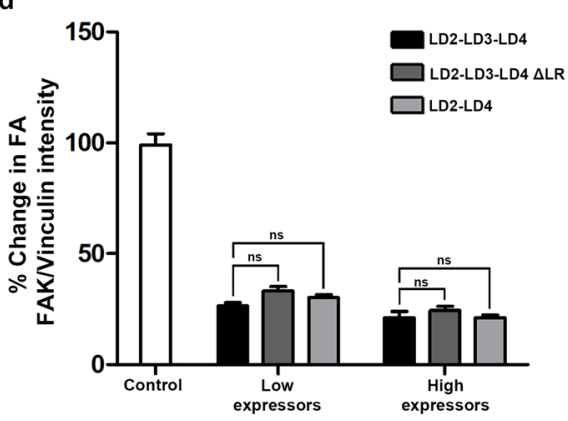

b

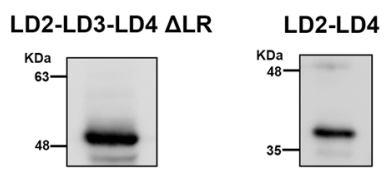

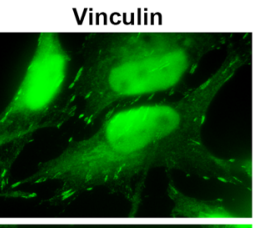
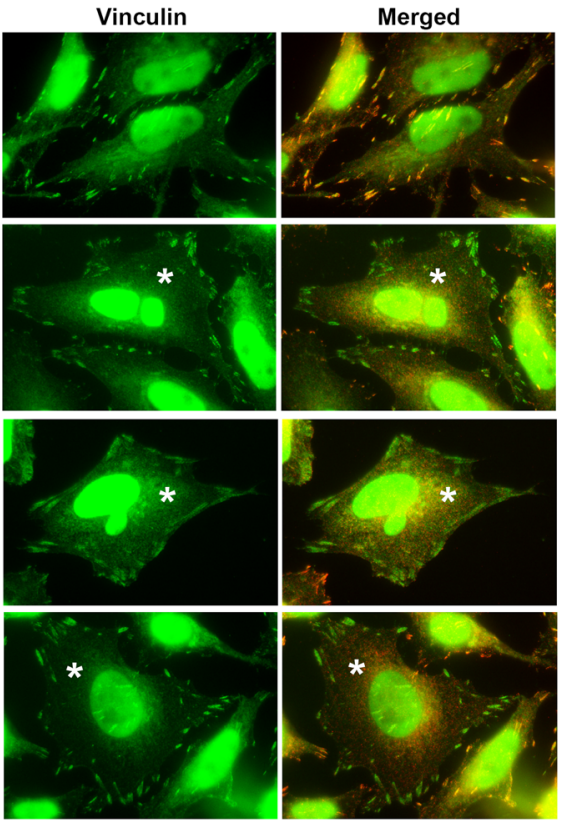

e

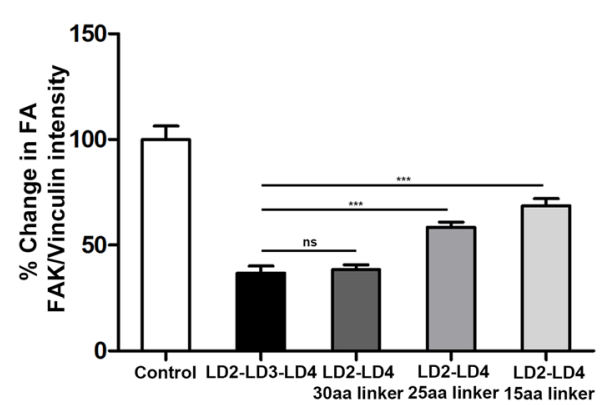

Fig. 7 The LD2 and LD4 motifs of paxillin, are sufficient for displacement of FAK from FAs a) Schematic representation of LD2-LD3-LD4 $\Delta$ LR, composed of amino acids 139-279 of Paxillin fused to GFP; and LD2-LD4, composed of LD2 and LD4 motifs of paxillin, amino acids 139-162 and 261-279 respectively, joined together by a 30 amino acid-long flexible linker and fused to GFP. b) Representative Western Blot showing expression of stable proteins, encoding GFP fused LD2-LD3-LD4 $\Delta$ LR and LD2-LD4, in HeLa cells (expected molecular weight $\sim 44$ kDa and $~ 34$ kDa respectively). c) Widefield images of HeLa cells, control or transiently transfected with GFP-fused LD2-LD3-LD4, LD2-LD3-LD4 $\Delta$ LR or LD2-LD4 and immunostained for FAK and Vinculin. Expressing cells are marked with an asterisk. Control cells display strong localization of FAK at FAs unlike cells expressing GFP-fused LD2-LD3-LD4, LD2-LD3-LD4 $\Delta$ LR or LD2-LD4. d) Quantification of the \% change in the mean FAKNinculin intensity at FAs in control cells (100 $\pm 4.56, n=335$ FAs from control cells) and cells expressing GFP LD2-LD3-LD4 (26.33 $\pm 1.46, n=163$ FAs from 16 low-expressing and 21.2 $\pm 2.73, n=147 \mathrm{FAs}$ from 15 high-expressing cells), GFP LD2-LD3-LD4 $\Delta$ LR $(33.04 \pm 2, n=150 \mathrm{FAs}$ from 16 lowexpressing and $24.32 \pm 1.84, n=133$ FAs from 14 high-expressing cells) or GFP LD2-LD4 (30 $\pm 1.3, n=140$ FAs from 15 low-expressing and $21.22 \pm$ 1.22, $n=225$ FAs from 17 high-expressing cells). Both GFP LD2-LD3-LD4 $\Delta$ LR and GFP LD2-LD4 displace FAK from FAs in a dose dependent manner and as efficiently as GFP LD2-LD3-LD4 does. e) Quantification of the \% change in the mean FAKNinculin intensity at FAs in control cells (100 $\pm 6.5, n=119$ FAs from 15 cells) and cells expressing GFP LD2-LD3-LD4 (36.65 \pm 3.4, n=140 FAs from 14 cells), or GFP LD2-LD4 with either a 30 amino acid linker ( $38.3 \pm 2.35, n=100$ FAs from 14 cells), 25 amino acid linker (58.47 $\pm 2.44, n=117$ FAs from 15 cells) or a 15 amino acid linker $(68.69 \pm 3.31, n=110$ FAs from 15 cells) It is evident that the 30 amino acid long linker displays equivalent efficiency to displace FAK from FAs to LD2-LD3-LD4. Scale bars $10 \mu \mathrm{m}$. The error bars represent standard error of the mean (S.E.M). ${ }^{* * *} ; p<0.0001$ 
linker was performed by evaluating the efficiency of LD2- GGGGSn-LD4 polypeptides, containing linkers of different sizes (15, 25 and 30 amino acids), to displace FAK from FAs (Fig. 7e). At the end, a 30 amino acidlong linker containing 6 (GGGGS) repeats was selected, leading to the generation of a new construct, hereunto referred to as LD2-LD4 (Fig. 7a and b). We went on to quantify the ability of LD2-LD4 to displace FAK from FAs in comparison to the original construct. As shown, LD2-LD4 is as efficient as the original in displacing FAK from FAs (Fig. 7c and d), suggesting that the sequence of the intermediate (LD2-LD4) linking region and LD3 are not essential for binding to FAK. The new polypeptide lacks critical phosphorylation sites present in the original and is devoid of any paxillin sequences other than the two LD motifs, ensuring improved specificity. Importantly the 30 amino acid linker is significantly shorter than the 99 amino acid linker contained in the original polypeptide; coupled with the removal of LD2 upstream sequences, this peptide is much smaller, only 69 amino acids $(6 \mathrm{kDa})$, compared to the original peptide that was 226 amino acids $(24 \mathrm{kDa})$ and thus well within the limits of solid-phase peptide synthesis. This effectively raises the possibility of using a synthetic polypeptide as an anti-metastatic agent.

\section{Inducible expression of LD2-LD4 interferes with the interaction of FAK with endogenous paxillin}

Having determined the minimum LD motif and linker length requirements of the polypeptide, we decided to confirm the molecular mechanism of action, which we postulated is the disruption of interactions between endogenous paxillin and FAK. Use of a transient expression system imposes limitations on using a biochemical approach, given the inability to attain $100 \%$ efficiency within a single transfection, uneven expression levels between transfected cells and variation in efficiency between transfections; we thus generated a stable HeLa cell line to inducibly express LD2-LD4 using a lentiviral vector system. As indicated in Fig. 8a, induction using Doxycycline, leads to the expression of a stable polypeptide at the expected molecular weight $(\sim 35 \mathrm{kD})$, which interacts with FAK, similarly to the transiently expressed polypeptide (Fig. 8b). Furthermore, inducible expression of LD2-LD4 led to clear displacement of FAK from FAs, while Vinculin localization (used as an FA marker) was unaffected as expected (Fig. 8c). Quantification of the FAK to Vinculin ratio showed that the inducible expression of LD2-LD4 displaced FAK with the same efficiency as the transiently expressed peptide (Fig. $8 \mathrm{~d}$ ). To validate the IF results we performed biochemical fractionation to isolate FAs followed by Western blot analysis, so as to determine resident protein levels. As shown in Fig. 8e the levels of FAK at FAs are markedly reduced, upon inducible expression of the LD2-LD4 polypeptide, and this is further supported by quantification of FAK/Paxillin ratio (both normalized to actin expression levels) (Fig. 8e). In contrast, Vinculin and paxillin levels are increased, in agreement with the immunofluorescence results described above. Collectively, these results provide firm confirmation that LD2-LD4 expression displaces FAK from FAs.

As extensively discussed in previously published work (from our group and others), targeting of FAK to FAs depends on interaction of the LD motifs of endogenous paxillin with the FAT domain of FAK. We postulated that the overexpressed LD2-LD4 polypeptide, shown to bind FAK (Fig. 1d and S1e), interferes with the ability of FAK to bind endogenous paxillin and therefore prevents FA targeting. To confirm this, we performed coimmunoprecipitation experiments to isolate FAK and co-precipitated proteins, from extracts of induced Hela cells that stably express LD2-LD4 (uninduced Hela cells as well as GFP expressing cells were used as controls). As shown in Fig. 8f, the levels of co-precipitated Paxillin in induced cells are markedly reduced compared to control cells. These data clearly show that expression of the polypeptide disrupts the interaction of FAK with endogenous paxillin, thus confirming the molecular mechanism of action.

\section{Dimers of a single LD motif can effectively displace FAK from FAs and reduce tumor cell migration}

The polypeptide used throughout this study consists of two separate LD motifs that have different sequences. However, designing and delivering a small molecule inhibitor consisting of two molecules would be quite challenging and complicated. We thus went on to examine the possibility that a single LD motif could bind to both HPs of the FAT domain and displace FAK.

We therefore proceeded to generate two new constructs encoding either LD2 or LD4 fused to GFP, hereunto referred to as GFP LD2 and GFP LD4 respectively (Fig. 9a) and examined the efficiency of the stable polypeptides expressed (Fig. 9b), to displace FAK from FAs. LD2 has been shown to bind to both HPs with equally high affinity, whereas LD4 only binds HP1 with high affinity, thus we expected that GFP LD2 would be more efficient in displacing FAK compared to GFP LD4 [43, 58, 59]. However, both LD2 and LD4 as monomers failed to displace FAK from FAs (Fig. $9 \mathrm{c}$ and d). These results, are in agreement with previous studies showing that peptides containing both LD2 and LD4 display higher affinity for FAT compared to single LDs [31, 43, $58,60]$, as well as the results discussed earlier, showing that LD2 and LD4 have to be connected through a flexible linker of the proper size in order to displace FAK 
a

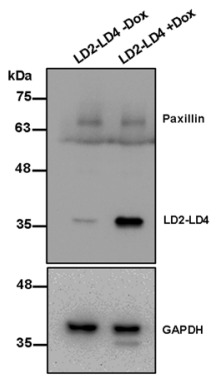

b

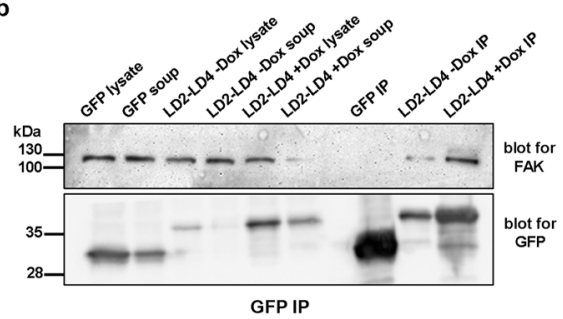

d
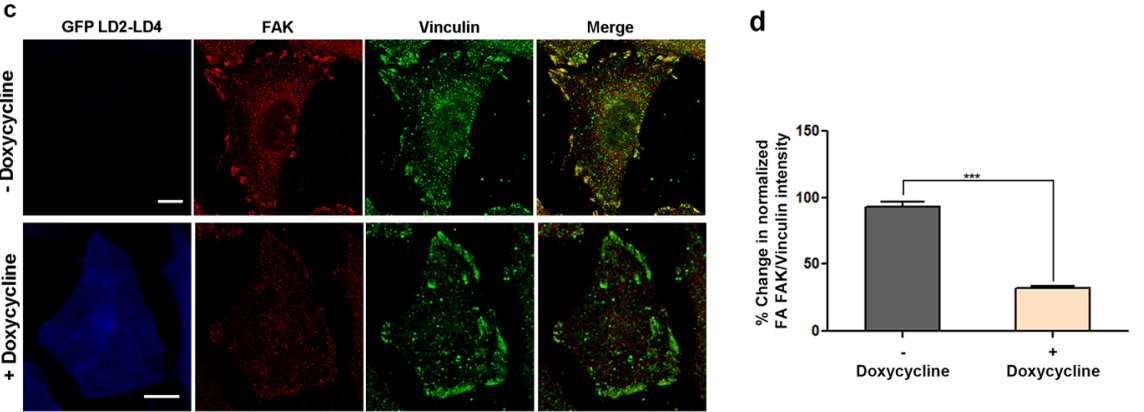
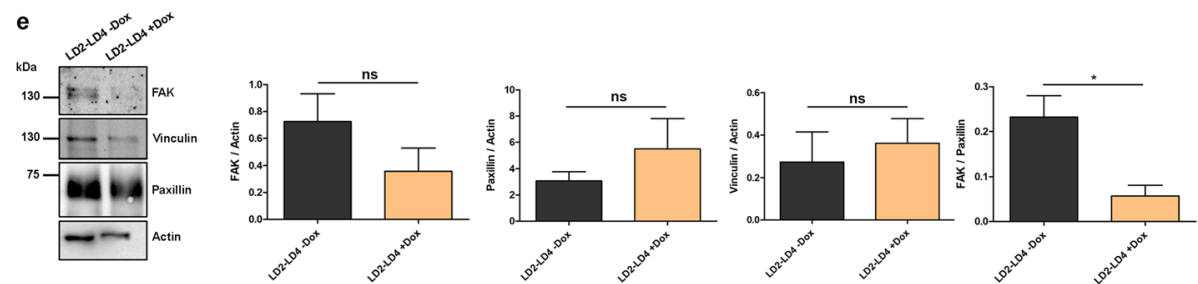

f

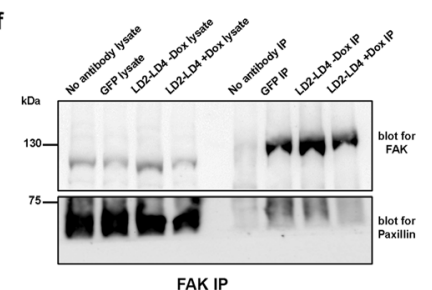

Fig. 8 LD2-LD4 expression interferes with the interaction between FAK and paxillin. Representative Western Blot from inducible GFP LD2-LD4 stable Hela cells indicating the expression levels of GFP LD2-LD4 compared to endogenous paxillin, upon induction with $10 \mu \mathrm{g} / \mathrm{ml}$ doxycycline for $24 \mathrm{~h}$. b) Western blot showing immunoprecipitated GFP, from Hela cells stably expressing GFP or GFP LD2-LD4 (from inducible GFP LD2-LD4 Hela cells, induced or not with $10 \mathrm{\mu g} / \mathrm{ml}$ doxycycline for $24 \mathrm{~h}$ ), blotted for GFP and FAK. FAK co-precipitation is observed in cells inducibly expressing GFP LD2-LD4. c) Confocal images of PFA-fixed inducible GFP LD2-LD4 Hela cells, immunostained for FAK and Vinculin. Expression of GFP LD2-LD4 upon treatment with $10 \mathrm{\mu g} / \mathrm{ml}$ doxycycline for $24 \mathrm{~h}$ (bottom panel) leads to decrease in the intensity of FAK at FAs, unlike Vinculin. d) Quantification of the \% change in the mean FAKNinculin intensity at FAs reveals $\sim 67 \%$ reduction in the FA-localized FAK in cells inducibly expressing GFP LD2-LD4 (32.28 $\pm 1.664 n=197$ FAs from 27 cells) compared to control non-induced cells (100 $\pm 4.01 n=211$ FAs from 28 cells). e) Representative western blots from isolated FAs of inducible GFP LD2-LD4 Hela cells, non-induced (left lane) or induced with $10 \mu \mathrm{g} / \mathrm{ml}$ doxycycline for $24 \mathrm{~h}$, blotted for FAK, Paxillin and Vinculin. Quantification of FAK/Actin $(0.7252 \pm 0.2068$ in non-induced, $0.3583 \pm 0.1684$ in induced cells), Paxillin/Actin (3.075 \pm 0.7076 in non-induced, $5.484 \pm 2.322$ in induced) and Vinculin/Actin (0.2735 \pm 0.1407 in non-induced, $0.3610 \pm 0.1156$ in induced) ratios, show reduction of FAK at the FAs of induced cells, unlike Paxillin and Vinculin which display increased FA-localization, verifying the results of IF experiments. This is further confirmed by quantification of the normalized FAK/Paxillin ratio in induced $(0.05682 \pm 0.02397)$ and non-induced $(0.2320 \pm 0.04788)$ cells, indicating $75 \%$ reduction in FA-localized FAK in cells expressing GFP LD2-LD4. f) Western blot of immunoprecipitated FAK from induced ( $24 \mathrm{~h}$ treatment with $10 \mathrm{\mu g} / \mathrm{ml}$ doxycycline) and non-induced GFP LD2-LD4 Hela cells, blotted for FAK and paxillin. The amount of co-precipitated paxillin is reduced in induced cells expressing GFP LD2-LD4. Non-induced GFP LD2-LD4 cells incubated with no antibody were used as negative control. Scale bars $10 \mu \mathrm{m}$. The error bars represent standard error of the mean (S.E.M). $* ; p<0.0 .05$ 
a

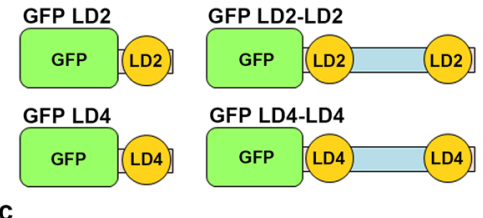

C
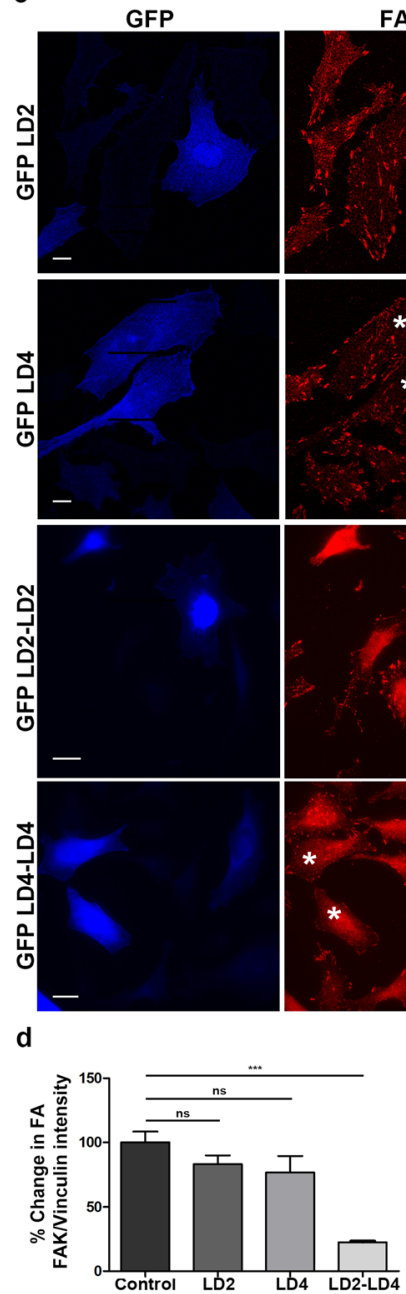

FAK
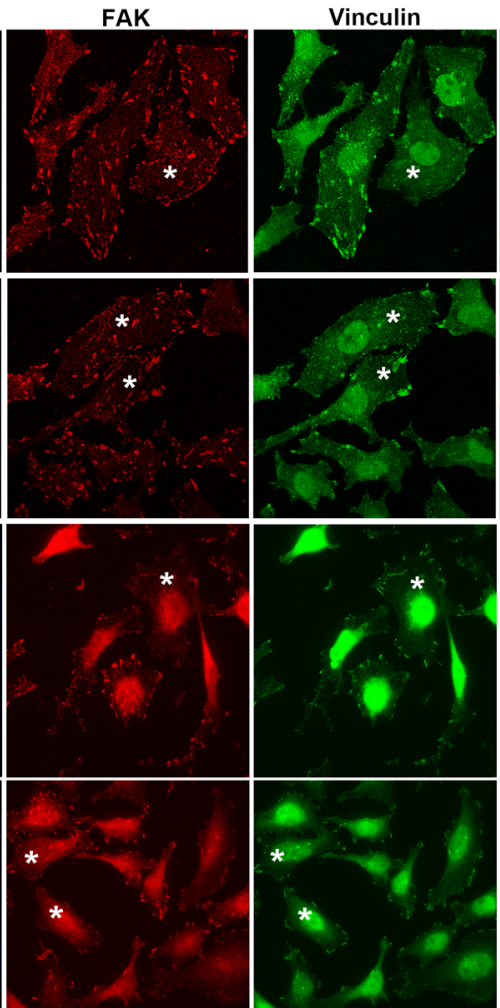

e

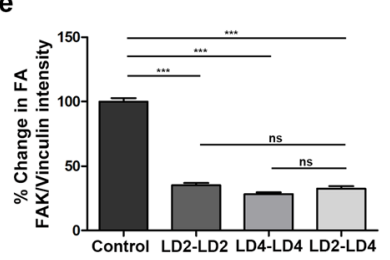

Fig. 9 Both FAK HPs can be effectively targeted with a single LD motif in the form of a dimer. a) Schematic representation of LD2 (composed of 420-440 amino acids of paxillin), LD4 (composed of 783-845 amino acids of paxillin), LD2-LD2 (composed of two LD2 motifs linked together through a flexible 30 amino acid linker) and LD4-LD4 (composed of two LD4 motifs linked together through a flexible 30 amino acid linker) constructs, fused to GFP. b) Representative Western Blots showing expression of stable proteins encoding GFP fused LD2 and LD4 (expected molecular weight $\sim 31 \mathrm{kDa}$ ) in the left panel and GFP fused LD2-LD2 and LD4-LD4 (expected molecular weight $~ 35 \mathrm{kDa}$ ) in the right panel. c) Widefield images of HeLa cells, transiently transfected with GFP-fused LD2, LD4, LD2-LD2 or LD4-LD4 and immunostained for FAK and Vinculin. Expressing cells are marked with an asterisk. GFP LD2 and GFP LD4 expressing cells display strong localization of FAK at FAs, similarly to control cells. GFP LD2-LD2 and GFP LD4-LD4 expressing display reduction in FA-localized FAK, compared to control cells. d) Quantification of the \% change in the mean FAKVinculin intensity at FAs in control cells (100 $\pm 8.43, n=388$ FAs from 25 cells) and cells expressing GFP LD2 (83 \pm 6.97 , $n=276$ FAs from 25 cells), GFP LD4 (76.54 $\pm 12.88, n=441$ FAs from 32 cells) or GFP LD2-LD4 (22.51 $\pm 0.9, n=406$ FAs from 30 cells). Neither GFP LD2 nor GFP LD4 displace FAK from FAs, unlike GFP LD2-LD4. e) Quantification of the \% change in the mean FAKNinculin intensity at FAs in control cells ( $100 \pm 2.64, n=542$ FAs from 30 cells) and cells expressing GFP LD2-LD2 (35.18 $\pm 1.75, n=334$ FAs from 27 cells), GFP LD4-LD4 (28.37 $\pm 1.31, n=322$ FAs from 28 cells) or GFP LD2-LD4 (32.61 $\pm 1.76, n=276$ FAs from 27 cells). Both GFP LD2-LD2 and GFP LD4-LD4 displace FAK from FAs, as efficiently as GFP LD2-LD4. f) Expression of GFP LD2-LD2 or GFP LD4-LD4 leads to decrease in the migration rate of MDA MB231 cells $(57.99 \pm 4.87, n=73$ cells expressing GFP LD2-LD4 and 37.52 $\pm 4.54, n=33$ cells expressing GFP LD4-LD4, compared to 100.0 $\pm 4.038, n=$ 89 control cells), similarly to GFP LD2-LD4 does $(49.84 \pm 5.43, n=49$ cells). Scale bars: $10 \mu \mathrm{m}$. The error bars represent standard error of the mean (S.E.M). ${ }^{* * *} ; p<0.0001$ 
from FAs. Taken together, these data provide strong evidence that an LD motif dimer is required for a high affinity interaction with FAK to take place.

To test this possibility and at the same time determine if a single LD motif could in fact target both HPs, we generated two new constructs encoding LD2-LD2 or LD4-LD4, separated by the optimized 30 amino acid linker described earlier (Fig. 9a and b). As shown (Fig. 9c-e) both LD2-LD2 and LD4-LD4 effectively displace FAK from FAs, with a similar efficiency as LD2-LD4 suggesting that both LD2 and LD4 can bind both HPs. Importantly, expression of either polypeptide reduced the migratory capacity of MDA MB-231 cells, as efficiently as LD2-LD4 (Fig. 9f).

These results lead to the conclusion that the function of FAK, specifically at FAs, can be efficiently targeted using the above described strategy, as long as the peptide topology is maintained (two LD motifs connected with an appropriate linker). More importantly, it suggests that a single small molecule mimic could potentially bind both HPs eliminating the need for two individual molecules.

\section{Discussion}

Both kinase-dependent and kinase-independent scaffolding functions of FAK are implicated in tumor development and metastasis and have previously been targeted, separately, by specific inhibitors. In this study, we present a novel strategy that can target and effectively block both adaptor and enzymatic functions of FAK at FAs, the major sites of FAK activity. The strategy relies on competing with interactions with physiological binding partners essential for FAK's FA localization, effectively displacing FAK from these complexes. We generated a polypeptide containing the Paxillin LD2LD3-LD4 motifs, which binds two hydrophobic pockets within the FAK FAT domain, thus preventing interactions with paxillin, previously described to be necessary for FA localization. Given that the polypeptide lacks the Paxillin localization determinants (LIM domains) it promotes FAK displacement from FAs. This is, to our knowledge, the first time an exogenously introduced molecule, is shown to prevent FAK FA localization, in a controlled, dose-dependent manner.

In contrast, when we tested $\mathrm{C} 4$, an inhibitor designed to block FAK-VEGFR3 interactions and reported to displace FAK from FAs $[17,47]$, we found that it failed to interfere with FA targeting of FAK, even at high concentrations. Given previous work showing that $\mathrm{C} 4$ acts through interactions with His 1025 on Helix 4 of the FAK FAT domain, we hypothesized that it may sterically hinder access to HP1. However, our data suggest that this inhibitor might not prevent interactions at HP1 or that interactions at both HPs need to be blocked, in order to displace FAK from FAs. This was also suggested by previous work showing that FAK mutants in which paxillin binding is completely abrogated by disruption of both HPs cannot localize to focal adhesions whereas FAK mutants that retain at least one functional HP (either HP1 or HP2), can still be successfully targeted to focal adhesions [33, 43].

Despite effective FAK displacement from FAs, localization of core FA proteins, including Talin, Integrins, Vinculin and Tensin was unaffected, suggesting that LD2-LD3-LD4 effects are FAK-specific, leaving FA composition broadly unchanged. It is important to note that these effects are observed on mature FAs and not nascent adhesions, where FAK has been proposed to promote the recruitment of talin [61]. Possible elimination of Talin from nascent adhesions by LD2-LD3-LD4 could potentially contribute to the migration defects observed. More importantly, the LD2-LD3-LD4 induced displacement of FAK from FAs has clear consequences on both FAK's kinase-dependent functions, including activation and downstream integrin signaling, as well as kinaseindependent scaffolding functions. Firstly, we show significant reduction of FAK phosphorylation at both Tyr 397 and Tyr576. Since autophosphorylation of Tyr397 is a direct consequence of FAK clustering within FA complexes [62] and given that LD2-LD3-LD4 displaces FAK from FAs, autophosphorylation is significantly reduced. Consequently, unphosphorylated Tyr397 can no longer support Src binding leading to reduced Src recruitment at FAs and Tyr576 phosphorylation [37, 50, 63, 64]. In addition, expression of LD2-LD3-LD4 leads to reduced Paxillin phosphorylation, one of the major FAK/Src downstream targets, but also to dramatically reduced levels of total phosphotyrosine at FAs. Tyrosine phosphorylation is the major signal transduction mechanism from FAs, therefore the observed reduction, suggests that LD2-LD3-LD4 expression, blocks integrin signaling via displacement of FAK from FA complexes and inhibition of downstream target phosphorylation. Therefore, LD2-LD3-LD4 can efficiently inhibit FAK's enzymatic activity and downstream signal transduction events without directly targeting its catalytic domain; instead it competes with endogenous paxillin, thus preventing FAK's targeting to FAs, which is essential for activation. Moreover, the LD2-LD3-LD4 induced displacement of FAK from FAs, also displaces p130Cas, an adaptor protein recruited to these multi-protein complexes through FAK. This suggests that our approach also targets and inhibits FAK's kinase-independent scaffolding functions, providing a distinct advantage over existing inhibitors.

FAK has a prominent role in FA assembly and disassembly, processes inherently linked to cell migration and metastasis. Expression of LD2-LD3-LD4 leads to significant defects in cell spreading and FA turnover, 
resembling the FAK-null fibroblast phenotype [53]. Consequently, we observed a clear, dose-dependent inhibition of 2D cell migration, suggesting that our approach effectively blocked FAK activity, in promoting cell movement. Despite the aforementioned defects, LD2-LD3-LD4 was well-tolerated, even when highly expressed, suggesting that binding is specific and nontoxic. This is yet another advantage over existing FAK kinase inhibitors, which have raised concerns with respect to toxicity due to limited specificity.

Perhaps more importantly than inhibiting cell migration, LD2-LD3-LD4 dramatically reduced the capacity of the highly metastatic MDA MB-231 cells to invade, in gel invasion assays. This suggests that this strategy for FAK inhibition has the potential to be further developed into an anti-metastatic agent, provided that efficient delivery can be accomplished. Our work points towards this direction, given that the only requirement to achieve effective FAK displacement from FAs is a $6 \mathrm{kD}$ polypeptide, comprised of an LD motif dimer (either LD2 or LD4 or a combination of the two), linked through a flexible, 30 amino-acid linker.

Current work focuses on a preclinical study to evaluate the strategy in vivo, using a mouse solid tumor model, as well as testing of synthetic peptide analogs and in silico molecular docking screens, to identify small molecule mimics capable of binding both HPs with high affinity. In addition, given the fact that both LD2 and LD4 can efficiently bind both HPs of the FAT domain of FAK (under the conditions described earlier), molecular dynamic simulations are currently under way, for the determination of an optimized sequence that could bind both HP1 and HP2 with improved affinity, that would possibly allow its use as a monomer.

The precise mechanism through which FAK is displaced from FAs in the presence of the peptide is not entirely clear, but several lines of evidence suggest that the two LD motifs on each polypeptide engage an individual FAT HP site on a single FAK molecule. Furthermore, the fact that the two LD motifs are linked probably enhances the avidity for this type of simultaneous binding. However, the possibility that the tandem LD motifs on the polypeptide link FAT domains from distinct FAK molecules, thus sequestering them away from FAs, cannot be excluded. This type of interaction is nonetheless highly unlikely given that previous studies using X-ray crystallography, solution NMR, and homology modeling, have shown the FAK-paxillin interaction to be mediated through binding of the LD2 and LD4 motifs of a single paxillin molecule to a single FAT domain [58]. In addition, our results, showing that for effective FAK displacement from FAs it is imperative that the two LD motifs of the polypeptide are linked through a flexible linker of a specific minimum length of 30 amino acids (shorter linkers of 15, 20 and 25 amino acids proved ineffective), also suggest that each polypeptide binds one FAK molecule. This experimentally determined optimal linker length is equivalent to $\sim 90 \AA$, and thus satisfies the requirement for sufficient length between two tandem LDs, so that the peptide can wrap around the FAT domain and allow simultaneous binding to the HPs of a single FAK molecule, in a parallel orientation, as previously proposed [58].

\section{Conclusion}

In conclusion, our data show that LD2-LD4 is a novel FAK inhibitor, that is well tolerated and functions by displacing the protein from FAs. This, as expected, results in the site-specific inhibition of FAK's kinase and scaffolding activities. Importantly, this peptide leads to impaired cell spreading, migration and invasion, raising the possibility that this promising new strategy can form the basis for the design of effective small-molecule FAK inhibitors, to prevent tumor metastasis.

\section{Supplementary Information}

Supplementary information accompanies this paper at https://doi.org/10. 1186/s12964-020-00671-1.

Additional file 1: Table S1. List of primers used for construct generation.

Additional file 2: Fig. S1. LD2-LD3-LD4 displaces both endogenous and exogenous FAK from FAs. Fig. S2. Inhibition of FAK Tyr397 phosphorylation prevents further activation of FAK without affecting its localization at FAs. Fig. S3. LD2-LD3-LD4 expression leads to displacement of FAK from FAs and reduction of the migratory capacity of tumor cells.

Additional file 3: Movie S1. Expression of LD2-LD3-LD4 reduces the migration of HeLa cells.

Additional file 4: Movie S2. Expression of LD2-LD3-LD4 inhibits the invasion of MDA MB-231 cells.

\section{Abbreviations}

FAK: Focal Adhesion Kinase; FAs: Focal Adhesions; FAT: Focal Adhesion Targeting; ECM: Extracellular Matrix; MMPs: Matrix Metalloproteinases; VEGF R: Vascular Endothelial Growth Factor Receptor-3; IGFR: Insulin-like Growth Factor Receptor; Mdm2: Mouse Double Minute 2; PI3K: Phosphoinositide 3 Kinase; HPs: Hydrophobic Pockets; LIM: Lin11, IsI-1 \& Mec-3; FRET: Förster Resonance Energy Transfer; 2-D: 2-Dimensional; 3-D: 3-Dimensional

\section{Acknowledgements}

We thank Dr. Andreas Constantinou (University of Cyprus) and Dr. Antonis Kirmizis (University of Cyprus) for kindly providing the H460 and HCT 116 cell lines respectively. We also thank Dr. Katerina Strari (University of Cyprus) for kindly providing the pLV-tetO-Oct4, pCMV-dR8.91 and pCMV-VGV-G plasmids.

\section{Authors' contributions}

IA and MK participated in the design of the study, performed the experiments, analysed the data and edited the manuscript. AC participated in the design of the study and drafted the manuscript. KK performed the experiments with LD motif dimers. AChr performed biochemical experiments on stable cell lines. MC generated the GFP and GFP LD2-LD4 stable cell lines. PS conceived the study, participated in its design and edited the manuscript. All authors read and approved the final manuscript. 


\section{Funding}

This work was co-funded by the European Regional Development Fund and the Republic of Cyprus through the Research and Innovation Foundation (Project: INFRASTRUCTURES/1216/0060).

\section{Availability of data and materials}

The data used and analyzed for the current study are available within the manuscript and its supplemental information files.

\section{Ethics approval and consent to participate}

Not applicable.

\section{Consent for publication}

Not applicable.

\section{Competing interests}

The authors declare that they have no competing interests.

Received: 14 May 2020 Accepted: 29 September 2020

Published online: 07 January 2021

\section{References}

1. Joyce JA, Pollard JW. Microenvironmental regulation of metastasis. Nat Rev Cancer. 2009;9(4):239-52.

2. Chatzizacharias NA, Kouraklis GP, Theocharis SE. Focal adhesion kinase: a promising target for anticancer therapy. Expert Opin Ther Targets. 2007; 11(10):1315-28.

3. Nagano M, Hoshino D, Koshikawa N, Akizawa T, Seiki M. Turnover of focal adhesions and cancer cell migration. Int J Cell Biol. 2012;2012:310616.

4. Petridou NI, Skourides PA. A ligand-independent integrin beta1 mechanosensory complex guides spindle orientation. Nat Commun. 2016;7:10899.

5. Petridou NI, Skourides PA. FAK transduces extracellular forces that orient the mitotic spindle and control tissue morphogenesis. Nat Commun. 2014;5:5240

6. Sulzmaier FJ, Jean C, Schlaepfer DD. FAK in cancer: mechanistic findings and clinical applications. Nat Rev Cancer. 2014;14(9):598-610.

7. Chen JS, Huang XH, Wang Q, Huang JQ, Zhang LJ, Chen XL, et al. Sonic hedgehog signaling pathway induces cell migration and invasion through focal adhesion kinase/AKT signaling-mediated activation of matrix metalloproteinase (MMP)-2 and MMP-9 in liver cancer. Carcinogenesis. 2013; 34(1):10-9.

8. McLean GW, Carragher NO, Avizienyte E, Evans J, Brunton VG, Frame MC. The role of focal-adhesion kinase in cancer - a new therapeutic opportunity. Nat Rev Cancer. 2005;5(7):505-15.

9. Zhao J, Guan JL. Signal transduction by focal adhesion kinase in cancer. Cancer Metastasis Rev. 2009;28(1-2):35-49.

10. Furuyama K, Doi R, Mori T, Toyoda E, Ito D, Kami K, et al. Clinical significance of focal adhesion kinase in resectable pancreatic cancer. World J Surg. 2006; 30(2):219-26.

11. Petridou NI, Stylianou P, Skourides PA. A dominant-negative provides new insights into FAK regulation and function in early embryonic morphogenesis. Development. 2013;140(20):4266-76.

12. Schwock J, Dhani N, Cao MP, Zheng J, Clarkson R, Radulovich N, et al. Targeting focal adhesion kinase with dominant-negative FRNK or Hsp90 inhibitor 17-DMAG suppresses tumor growth and metastasis of SiHa cervical xenografts. Cancer Res. 2009;69(11):4750-9.

13. Duxbury MS, Ito H, Zinner MJ, Ashley SW, Whang EE. Focal adhesion kinase gene silencing promotes anoikis and suppresses metastasis of human pancreatic adenocarcinoma cells. Surgery. 2004;135(5):555-62

14. Duxbury MS, Ito H, Benoit E, Zinner MJ, Ashley SW, Whang EE. RNA interference targeting focal adhesion kinase enhances pancreatic adenocarcinoma gemcitabine chemosensitivity. Biochem Biophys Res Commun. 2003;311(3):786-92.

15. Lv PC, Jiang AQ, Zhang WM, Zhu HL. FAK inhibitors in Cancer, a patent review. Expert Opin Ther Pat. 2018;28(2):139-45.

16. Golubovskaya VM, Figel S, Ho BT, Johnson CP, Yemma M, Huang G, et al. A small molecule focal adhesion kinase (FAK) inhibitor, targeting Y397 site: 1(2-hydroxyethyl)-3, 5, 7-triaza-1-azoniatricyclo [3.3.1.1(3,7)]decane; bromide effectively inhibits FAK autophosphorylation activity and decreases cancer cell viability, clonogenicity and tumor growth in vivo. Carcinogenesis. 2012; 33(5):1004-13.

17. Kurenova EV, Hunt DL, He D, Magis AT, Ostrov DA, Cance WG. Small molecule chloropyramine hydrochloride (C4) targets the binding site of focal adhesion kinase and vascular endothelial growth factor receptor 3 and suppresses breast cancer growth in vivo. J Med Chem. 2009:52(15):4716-24.

18. Liu W, Bloom DA, Cance WG, Kurenova EV, Golubovskaya VM, Hochwald SN. FAK and IGF-IR interact to provide survival signals in human pancreatic adenocarcinoma cells. Carcinogenesis. 2008;29(6):1096-107.

19. Ucar DA, Magis AT, He DH, Lawrence NJ, Sebti SM, Kurenova E, et al. Inhibiting the interaction of CMET and IGF-1R with FAK effectively reduces growth of pancreatic cancer cells in vitro and in vivo. Anti Cancer Agents Med Chem. 2013;13(4):595-602.

20. Ucar DA, Kurenova E, Garrett TJ, Cance WG, Nyberg C, Cox A, et al. Disruption of the protein interaction between FAK and IGF-1R inhibits melanoma tumor growth. Cell Cycle. 2012;11(17):3250-9.

21. Golubovskaya VM, Palma NL, Zheng M, Ho B, Magis A, Ostrov D, et al. A small-molecule inhibitor, 5'-O-tritylthymidine, targets FAK and Mdm-2 interaction, and blocks breast and colon tumorigenesis in vivo. Anti Cancer Agents Med Chem. 2013;13(4):532-45.

22. Golubovskaya VM, Ho B, Zheng M, Magis A, Ostrov D, Morrison C, et al. Disruption of focal adhesion kinase and p53 interaction with small molecule compound R2 reactivated p53 and blocked tumor growth. BMC Cancer. 2013;13:342.

23. Brown MC, Curtis MS, Turner CE. Paxillin LD motifs may define a new family of protein recognition domains. Nat Struct Biol. 1998;5(8):677-8.

24. Brown MC, Perrotta JA, Turner CE. Identification of LIM3 as the principal determinant of paxillin focal adhesion localization and characterization of a novel motif on paxillin directing vinculin and focal adhesion kinase binding. J Cell Biol. 1996:135(4):1109-23.

25. Schaller MD, Otey CA, Hildebrand JD, Parsons JT. Focal adhesion kinase and paxillin bind to peptides mimicking beta integrin cytoplasmic domains. J Cell Biol. 1995;130(5):1181-7.

26. Schlaepfer DD, Hanks SK, Hunter T, van der Geer P. Integrin-mediated signal transduction linked to Ras pathway by GRB2 binding to focal adhesion kinase. Nature. 1994;372(6508):786-91.

27. Harte MT, Hildebrand JD, Burnham MR, Bouton AH, Parsons JT. p130Cas, a substrate associated with v-Src and v-Crk, localizes to focal adhesions and binds to focal adhesion kinase. J Biol Chem. 1996;271(23):13649-55.

28. Liu Y, Loijens JC, Martin KH, Karginov AV, Parsons JT. The association of ASAP1, an ADP ribosylation factor-GTPase activating protein, with focal adhesion kinase contributes to the process of focal adhesion assembly. Mol Biol Cell. 2002;13(6):2147-56.

29. Guinebault C, Payrastre B, Racaud-Sultan C, Mazarguil H, Breton M, Mauco G, et al. Integrin-dependent translocation of phosphoinositide 3-kinase to the cytoskeleton of thrombin-activated platelets involves specific interactions of p85 alpha with actin filaments and focal adhesion kinase. J Cell Biol. 1995; 129(3):831-42

30. Hayashi I, Vuori K, Liddington RC. The focal adhesion targeting (FAT) region of focal adhesion kinase is a four-helix bundle that binds paxillin. Nat Struct Biol. 2002:9(2):101-6.

31. Hoellerer MK, Noble ME, Labesse G, Campbell ID, Werner JM, Arold ST. Molecular recognition of paxillin LD motifs by the focal adhesion targeting domain. Structure. 2003;11(10):1207-17.

32. Arold ST, Hoellerer MK, Noble ME. The structural basis of localization and signaling by the focal adhesion targeting domain. Structure. 2002;10(3):319-27.

33. Deramaudt TB, Dujardin D, Noulet F, Martin S, Vauchelles R, Takeda K, et al. Altering FAK-paxillin interactions reduces adhesion, migration and invasion processes. PLoS One. 2014;9(3):e92059.

34. Antoniades I, Stylianou P, Christodoulou N, Skourides PA. Addressing the functional determinants of FAK during Ciliogenesis in multiciliated cells. J Biol Chem. 2017;292(2):488-504.

35. Shen $Y$, Schaller MD. Focal adhesion targeting: the critical determinant of FAK regulation and substrate phosphorylation. Mol Biol Cell. 1999;10(8): 2507-18.

36. Brown MC, Perrotta JA, Turner CE. Serine and threonine phosphorylation of the paxillin LIM domains regulates paxillin focal adhesion localization and cell adhesion to fibronectin. Mol Biol Cell. 1998:9(7):1803-16.

37. Calalb MB, Polte TR, Hanks SK. Tyrosine phosphorylation of focal adhesion kinase at sites in the catalytic domain regulates kinase activity: a role for Src family kinases. Mol Cell Biol. 1995;15(2):954-63. 
38. Cary LA, Chang JF, Guan JL. Stimulation of cell migration by overexpression of focal adhesion kinase and its association with Src and Fyn. J Cell Sci. 1996;109(Pt 7):1787-94.

39. Stadtfeld M, Maherali N, Breault DT, Hochedlinger K. Defining molecular cornerstones during fibroblast to iPS cell reprogramming in mouse. Cell Stem Cell. 2008;2(3):230-40.

40. Antoniades I, Stylianou P, Skourides PA. Making the connection: ciliary adhesion complexes anchor basal bodies to the actin cytoskeleton. Dev Cell. 2014;28(1):70-80.

41. Campeau E, Ruhl VE, Rodier F, Smith CL, Rahmberg BL, Fuss JO, et al. A versatile viral system for expression and depletion of proteins in mammalian cells. PLoS One. 2009;4(8):e6529.

42. Jones MC, Humphries JD, Byron A, Millon-Fremillon A, Robertson J, Paul NR, et al. Isolation of integrin-based adhesion complexes. Curr Protoc Cell Biol. 2015:66:9 $81-9815$

43. Scheswohl DM, Harrell JR, Rajfur Z, Gao G, Campbell SL, Schaller MD. Multiple paxillin binding sites regulate FAK function. J Mol Signal. 2008;3:1

44. Jagadeeswaran $R$, Surawska H, Krishnaswamy S, Janamanchi V, Mackinnon AC, Seiwert TY, et al. Paxillin is a target for somatic mutations in lung cancer: implications for cell growth and invasion. Cancer Res. 2008;68(1): $132-42$.

45. Kawada I, Hasina R, Lennon FE, Bindokas VP, Usatyuk P, Tan YH, et al. Paxillin mutations affect focal adhesions and lead to altered mitochondrial dynamics: relevance to lung cancer. Cancer Biol Ther. 2013;14(7):679-91.

46. Neerathilingam M, Bairy SG, Mysore S. Deciphering mode of action of functionally important regions in the intrinsically disordered Paxillin (residues 1-313) using its interaction with FAT (focal adhesion targeting domain of focal adhesion kinase). PLoS One. 2016;11(2):e0150153.

47. Stewart JE, Ma X, Megison M, Nabers H, Cance WG, Kurenova EV, et al. Inhibition of FAK and VEGFR-3 binding decreases tumorigenicity in neuroblastoma. Mol Carcinog. 2015;54(1):9-23.

48. Owen JD, Ruest PJ, Fry DW, Hanks SK. Induced focal adhesion kinase (FAK) expression in FAK-null cells enhances cell spreading and migration requiring both auto- and activation loop phosphorylation sites and inhibits adhesion-dependent tyrosine phosphorylation of Pyk2. Mol Cell Biol. 1999; 19(7):4806-18

49. Zaidel-Bar R, Milo R, Kam Z, Geiger B. A paxillin tyrosine phosphorylation switch regulates the assembly and form of cell-matrix adhesions. J Cell Sci. 2007;120(Pt 1):137-48.

50. Schaller MD, Hildebrand JD, Shannon JD, Fox JW, Vines RR, Parsons JT. Autophosphorylation of the focal adhesion kinase, pp125FAK, directs SH2dependent binding of pp60src. Mol Cell Biol. 1994;14(3):1680-8.

51. Wu JC, Chen YC, Kuo CT, Wenshin Yu H, Chen YQ, Chiou A, et al. Focal adhesion kinase-dependent focal adhesion recruitment of $\mathrm{SH} 2$ domains directs SRC into focal adhesions to regulate cell adhesion and migration. Sci Rep. 2015;5:18476.

52. Slack-Davis JK, Martin KH, Tilghman RW, Iwanicki M, Ung EJ, Autry C, et al. Cellular characterization of a novel focal adhesion kinase inhibitor. J Biol Chem. 2007;282(20):14845-52.

53. Ilic D, Furuta Y, Kanazawa S, Takeda N, Sobue K, Nakatsuji N, et al. Reduced cell motility and enhanced focal adhesion contact formation in cells from FAK-deficient mice. Nature. 1995;377(6549):539-44.

54. Lim Y, Lim ST, Tomar A, Gardel M, Bernard-Trifilo JA, Chen XL, et al. PyK2 and FAK connections to p190Rho guanine nucleotide exchange factor regulate RhoA activity, focal adhesion formation, and cell motility. J Cell Biol. 2008;180(1):187-203.

55. Franco SJ, Huttenlocher A. Regulating cell migration: calpains make the cut. J Cell Sci. 2005;118(Pt 17):3829-38.

56. Edmondson R, Broglie JJ, Adcock AF, Yang L. Three-dimensional cell culture systems and their applications in drug discovery and cell-based biosensors. Assay Drug Dev Technol. 2014;12(4):207-18.

57. Yamaguchi H, Wyckoff J, Condeelis J. Cell migration in tumors. Curr Opin Cell Biol. 2005;17(5):559-64.

58. Bertolucci CM, Guibao CD, Zheng J. Structural features of the focal adhesion kinase-paxillin complex give insight into the dynamics of focal adhesion assembly. Protein Sci. 2005;14(3):644-52.

59. Gao G, Prutzman KC, King ML, Scheswohl DM, DeRose EF, London RE, et al. NMR solution structure of the focal adhesion targeting domain of focal adhesion kinase in complex with a paxillin LD peptide: evidence for a twosite binding model. J Biol Chem. 2004;279(9):8441-51.
60. Turner CE, Brown MC, Perrotta JA, Riedy MC, Nikolopoulos SN, McDonald AR, et al. Paxillin LD4 motif binds PAK and PIX through a novel 95-kD ankyrin repeat, ARF-GAP protein: a role in cytoskeletal remodeling. J Cell Biol. 1999;145(4):851-63.

61. Lawson C, Lim ST, Uryu S, Chen XL, Calderwood DA, Schlaepfer DD. FAK promotes recruitment of Talin to nascent adhesions to control cell motility. J Cell Biol. 2012;196(2):223-32.

62. Toutant M, Costa A, Studler JM, Kadare G, Carnaud M, Girault JA. Alternative splicing controls the mechanisms of FAK autophosphorylation. Mol Cell Biol. 2002;22(22):7731-43.

63. Calalb MB, Zhang X, Polte TR, Hanks SK. Focal adhesion kinase tyrosine-861 is a major site of phosphorylation by Src. Biochem Biophys Res Commun. 1996;228(3):662-8.

64. Schlaepfer DD, Hunter T. Evidence for in vivo phosphorylation of the Grb2 SH2-domain binding site on focal adhesion kinase by Src-family proteintyrosine kinases. Mol Cell Biol. 1996;16(10):5623-33.

\section{Publisher's Note}

Springer Nature remains neutral with regard to jurisdictional claims in published maps and institutional affiliations.
Ready to submit your research? Choose BMC and benefit from:

- fast, convenient online submission

- thorough peer review by experienced researchers in your field

- rapid publication on acceptance

- support for research data, including large and complex data types

- gold Open Access which fosters wider collaboration and increased citations

- maximum visibility for your research: over $100 \mathrm{M}$ website views per year

At BMC, research is always in progress.

Learn more biomedcentral.com/submissions 